\title{
Distinct Drimane Chemotypes in Tasmanian Mountain Pepper (Tasmannia lanceolata): Differences in the Profiles of Pungent Leaf Phytochemicals Associated with Altitudinal Cline
}

Bianca J. Deans, ${ }^{a}$ Jeremy Just, ${ }^{a}$ Laura Tedone,${ }^{a, b}$ Nathan L. Kilah, ${ }^{a}$ Gregory J. Jordan, ${ }^{c}$ Alex C. Bissember, ${ }^{a^{*}}$ Jason A. Smith ${ }^{a^{\star}}$

${ }^{a}$ School of Natural Sciences - Chemistry, University of Tasmania, Hobart, Tasmania, Australia ${ }^{b}$ Australian Centre for Research on Separation Science (ACROSS), University of Tasmania, Hobart, Tasmania, Australia

${ }^{c}$ School of Natural Sciences - Biology, University of Tasmania, Hobart, Tasmania, Australia

\section{Supporting Information}

\section{Table of Contents}

I. Materials \& Methods: Supplementary Information

II. Voucher Specimen Numbers \& GPS Location Data

III. Yield Data: Drimane Sesquiterpenoids (from Heptane Extracts)

IV. X-Ray Crystallographic Data

S-8

V. Representative Spectroscopic \& MS Data 


\section{Materials \& Methods: Supplementary Information}

\section{Extraction of $T$. lanceolata: Cradle Mountain (Site 1)}

PHWE of Bulk Site Sample. Fresh leaves collected from Cradle Mountain (site 1) were airdried at room temperature for 7 days. Following this period, dried $T$. lanceolata leaves (1.5 g) from each of the ten specimens (A1-J1) were then coarsely ground in a spice grinder, combined (total of $15 \mathrm{~g}$ leaf material from all ten specimens) and mixed with sand $(4 \mathrm{~g}$ ), placed into the portafilter (sample compartment) of an espresso machine and extracted using $35 \% \mathrm{v} / \mathrm{v} \mathrm{EtOH} / \mathrm{H}_{2} \mathrm{O}$ (200 $\mathrm{mL}$ of a hot solution). The ensuing extract was then concentrated under reduced pressure on a rotary evaporator to $\sim 125 \mathrm{~mL}$ to remove EtOH (no heating was applied during this process). The ensuing PHWE mixture was extracted with heptane $(4 \times 50 \mathrm{~mL})$. The organic fractions were then combined, dried $\left(\mathrm{MgSO}_{4}\right)$, filtered and the solvent removed under reduced pressure to provide a green residue $(79.4 \mathrm{mg})$. Analysis via ${ }^{1} \mathrm{H}$ NMR spectroscopy and TLC supported the presence of polygodial (1) as the single major drimane sesquiterpenoid.

Ethyl Acetate Extraction of Bulk Population Sample. Following the heptane solvent extraction, the remaining aqueous layer was extracted with EtOAc and ${ }^{1} \mathrm{H}$ NMR spectroscopic analysis was performed as described for samples from Site 4 (see Materials and Methods section).

PHWE of Individual Samples (A1-J1). Following the bulk extraction, each individual sample (A1-J1) was extracted in an identical method and quantities to that described for samples from Site 4 (see Materials and Methods section). The ensuing PHWE mixture was extracted with heptane $(4 \times 50 \mathrm{~mL})$. The organic fractions were then combined, dried $\left(\mathrm{MgSO}_{4}\right)$ and filtered. 1,3,5-trimethoxybenzene (internal standard, $5.00 \mathrm{~mL}$ of a $0.0213 \mathrm{M}$ solution in EtOH) was added and the solvent removed under reduced pressure to provide a green residue. The ${ }^{1} \mathrm{H}$ NMR spectrum $\left(\mathrm{CDCl}_{3}\right)$ of the crude extract thus enabled the yield of polygodial to be determined. The ratio of polygodial (1) to 1,3,5-trimethoxybenzene standard was determined by the averaged integration of the two polygodial aldehyde signals $(9.53 \& 9.45$ $\mathrm{ppm})$ relative to the singlet $(6.08 \mathrm{ppm})$ corresponding to the methine signal of $1,3,5-$ trimethoxybenzene. This extraction process and analysis was conducted for each of the ten samples, and summarized in Table 1. See Supporting Information Table S2 for the yields from individuals within this population.

\section{Extraction of T. lanceolata: Mt. Field (Site 2)}

PHWE of Bulk Sample. Fresh leaves collected from Mt. Field (site 2) were air-dried at room temperature for 7 days. Following this period, a portion the dried $T$. lanceolata leaves $(1.5 \mathrm{~g})$ from each of the nine specimens (A2-I2) were then coarsely ground in a spice grinder, combined together (total of $13.5 \mathrm{~g}$ leaf material from all specimens) and mixed with sand (4 $\mathrm{g}$ ), placed into the portafilter (sample compartment) of an espresso machine and extracted using $35 \% \mathrm{v} / \mathrm{v} \mathrm{EtOH} / \mathrm{H}_{2} \mathrm{O}$ (200 $\mathrm{mL}$ of hot solution). The ensuing extract was then concentrated under reduced pressure on a rotary evaporator to $\sim 125 \mathrm{~mL}$ to remove EtOH (no heating was applied during this process). The ensuing PHWE mixture was extracted with heptane $(4 \times 50 \mathrm{~mL})$. The organic fractions were then combined, dried $\left(\mathrm{MgSO}_{4}\right)$, filtered and the solvent removed under reduced pressure to provide a green residue (42 mg). Analysis via ${ }^{1} \mathrm{H}$ NMR spectroscopy and TLC of the crude extract indicated the presence of a 
major compound that was not previously observed within $T$. lanceolata extracts, so the crude extract $(42 \mathrm{mg})$ was purified by automated flash column chromatography $(0-40 \%$ EtOAc/hexanes; $14 \mathrm{~min} ; 4 \mathrm{~g}$ silica cartridge) to afford $1 \beta$-acetoxy-9-deoxyisomuzigadial (6) as a pale green film $(10.3 \mathrm{mg}, 0.067 \% \mathrm{w} / \mathrm{w})$, and $3 \beta$-acetoxydrimenin (11) as colorless crystals (3.4 mg, $0.023 \% \mathrm{w} / \mathrm{w})$.

Ethyl Acetate Extraction of Bulk Population Sample \& Purification. Following the heptane solvent extraction, the remaining aqueous layer was subjected to an EtOAc extraction, evaporated to dryness and subjected to ${ }^{1} \mathrm{H}$ NMR spectroscopic analysis identical to that described for samples from Site 4 (see Materials and Methods section). The resulting crude EtOAc extract $(674 \mathrm{mg})$ was adsorbed onto silica gel and purified by automated flash chromatography (0-20\% methanol/EtOAc; $14 \mathrm{~min} ; 12 \mathrm{~g}$ silica cartridge) to afford chlorogenic acid (23) as the major component as small colorless needles (216 mg, 1.4\% w/w).

PHWE of Individual Samples (A2-12). Following the bulk extraction, each individual sample (A2-12) was extracted in an identical method and quantities to that described for samples from Site 4 (see Materials and Methods section). The ensuing PHWE mixture was extracted with heptane $(4 \times 50 \mathrm{~mL})$. The organic fractions were then combined, dried $\left(\mathrm{MgSO}_{4}\right)$ and filtered. 1,3,5-trimethoxybenzene (internal standard, $5.00 \mathrm{~mL}$ of a $0.0213 \mathrm{M}$ solution in EtOH) was added and the solvent removed under reduced pressure to provide a green residue. The yield of the major component $1 \beta$-acetoxy-9-deoxyisomuzigadial (6) was determined via ${ }^{1} \mathrm{H}$ NMR spectroscopic analysis $\left(\mathrm{CDCl}_{3}\right)$ of the crude heptane extracts. The ratio of $1 \beta$ acetoxy-9-deoxyisomuzigadial (6) was determined by the averaged integration of the two aldehyde signals ( $9.39 \& 9.87 \mathrm{ppm}$ ) relative to the singlet $(6.08 \mathrm{ppm})$ corresponding to the methine signal of 1,3,5-trimethoxybenzene. This extraction process and analysis was conducted for each of the ten samples, and summarized in Table 1. See Supporting Information Table S2 for the yields from individuals within this population.

\section{Extraction of T. lanceolata: Mt. Wellington - Subalpine Woodland (Site 3)}

PHWE of Individual Samples (A3-K3). Fresh leaves collected from Mt. Wellington (site 3) were air-dried at room temperature for 7 days. Following this period, a portion of the dried $T$. lanceolata leaves $(1.5 \mathrm{~g})$ from each of the eleven specimens (A3-K3) were then coarsely ground in a spice grinder, combined (total of $16.5 \mathrm{~g}$ leaf material from all eleven specimens) and mixed with sand ( $4 \mathrm{~g}$ ), placed into the portafilter (sample compartment) of an espresso machine and extracted using $35 \% \mathrm{v} / \mathrm{v} \mathrm{EtOH} / \mathrm{H}_{2} \mathrm{O}(200 \mathrm{~mL}$ of a hot solution). The ensuing extract was then concentrated under reduced pressure on a rotary evaporator to $\sim 125 \mathrm{~mL}$ to remove $\mathrm{EtOH}$ (no heating was applied during this process). The ensuing PHWE mixture was extracted with heptane $(4 \times 50 \mathrm{~mL})$. The organic fractions were then combined, dried $\left(\mathrm{MgSO}_{4}\right)$, filtered and the solvent removed under reduced pressure to provide a green residue $(100 \mathrm{mg})$. Analysis via ${ }^{1} \mathrm{H}$ NMR spectroscopy and TLC analysis of the crude extract indicated the presence of $1 \beta$-acetoxy-9-deoxyisomuzigadial (6) as the major compound, in addition to a mixture of minor components, so the crude extract $(100 \mathrm{mg})$ was purified by automated flash chromatography (0-40\% EtOAc/hexanes; $7 \mathrm{~min} ; 4 \mathrm{~g}$ silica cartridge) to afford $1 \beta$-acetoxy-9-deoxyisomuzigadial (6) $(12.0 \mathrm{mg}, 0.073 \% \mathrm{w} / \mathrm{w})$ and $3 \beta$-acetoxydrimenin (11) $(9.3 \mathrm{mg}, 0.056 \% \mathrm{w} / \mathrm{w})$.

Ethyl Acetate Extraction of Bulk Population Sample. Following the heptane solvent extraction, the remaining aqueous layer was extracted with EtOAc and ${ }^{1} \mathrm{H} N M R$ 
spectroscopic analysis was performed as described for samples from Site 4 (see Materials and Methods section).

PHWE of Individual Samples (A3-K3). Following the bulk extraction, each individual sample (A3-K3) was extracted in an identical method and quantities to that described for samples from Site 4 (see Materials and Methods section). The ensuing PHWE mixture was extracted with heptane $(4 \times 50 \mathrm{~mL})$. The organic fractions were then combined, dried $\left(\mathrm{MgSO}_{4}\right)$ and filtered. 1,3,5-trimethoxybenzene (internal standard, $5.00 \mathrm{~mL}$ of a $0.0213 \mathrm{M}$ solution in $\mathrm{EtOH}$ ) was added and the solvent removed under reduced pressure to provide a green residue. The yield of $1 \beta$-acetoxy-9-deoxyisomuzigadial (6) was determined by ${ }^{1} \mathrm{H}$ NMR spectroscopic analysis via by the ratio of the averaged integration of the two aldehyde signals $(9.39 \& 9.87 \mathrm{ppm})$ relative to the integration of the singlet $(6.08 \mathrm{ppm})$ corresponding to the methine signal of 1,3,5-trimethoxybenzene. This extraction process and analysis was conducted for each of the eleven samples, and with ${ }^{1} \mathrm{H}$ NMR yield ranges summarized in Table 1. See Supporting Information (Table S2) for the yields from individuals within this population.

\section{PHWE of Commercial T. lanceolata}

Bronzewing Farm. Fresh leaves from two separate clonal female $T$. lanceolata populations ("A" and "B") were collected from a commercial farm. The leaves were air dried for 1 week, after which the dried $T$. lanceolata leaves $(15 \mathrm{~g})$ were coarsely ground in a spice grinder, mixed with sand $(4 \mathrm{~g})$, and were extracted in an identical method to that described for samples from Site 4 (see Materials and Methods section). The ensuing PHWE extracts were then treated to the identical extraction method described for samples from Site 4 (see Materials and Methods section). The internal standard 1,3,5-trimethoxybenzene (5.00 mL of a $0.0213 \mathrm{M}$ solution in $\mathrm{EtOH}$ ) was added and the solvent removed under reduced pressure to provide a green residue. Analysis of the crude extract via ${ }^{1} \mathrm{H}$ NMR spectrum $\left(\mathrm{CDCl}_{3}\right)$ indicated that polygodial (1) was the major component, with the yield of polygodial (1) determined by the averaged integration of the two aldehyde methine signals $(9.53 \& 9.45$ $\mathrm{ppm})$ relative to the singlet $(6.08 \mathrm{ppm})$ corresponding to the methine signal of $1,3,5-$ trimethoxybenzene. This process was conducted for each population respectively, with the yield data summarized in Table 1. Following the heptane solvent extraction, the remaining aqueous layer was extracted with EtOAc and ${ }^{1} \mathrm{H}$ NMR spectroscopic analysis was performed as described for samples from Site 4 (see Materials and Methods section).

Diemen Pepper. Pre-prepared dried and ground $T$. lanceolata leaves $(15 \mathrm{~g})$ were mixed with sand $(4 \mathrm{~g})$ and extracted via PHWE, heptane solvent extracted and analyzed in an identical method to that described for samples from Site 4 (see Materials and Methods section). ${ }^{1} \mathrm{H}$ NMR yields are summarized in Table 1. Following the heptane solvent extraction, the remaining aqueous layer was extracted with EtOAc and ${ }^{1} \mathrm{H}$ NMR spectroscopic analysis was performed as described for samples from Site 4 (see Materials and Methods section). 


\section{Voucher Specimen Numbers \& GPS Location Data}

Table S1A. T. lanceolata population sites \& voucher details.

\begin{tabular}{|c|c|c|c|c|}
\hline Population Site & Sample & Specimen ID (Population Voucher Plate \#) & GPS Location & Altitude (m) \\
\hline Cradle Mountain (Site 1) & A1 & BD-2018-01 (HO594860) & S $41^{\circ} 34.758^{\prime}$ E $145^{\circ} 56.183^{\prime}$ & 888 \\
\hline Cradle Mountain (Site 1) & B1 & BD-2018-02 (HO594860) & S $41^{\circ} 34.757^{\prime}$ E $145^{\circ} 56.191^{\prime}$ & 886 \\
\hline Cradle Mountain (Site 1) & C1 & BD-2018-03 (HO594860) & S $41^{\circ} 34.757^{\prime}$ E $145^{\circ} 56.182^{\prime}$ & 887 \\
\hline Cradle Mountain (Site 1) & D1 & BD-2018-04 (HO594860) & S $41^{\circ} 34.754^{\prime}$ E $145^{\circ} 56.197^{\prime}$ & 884 \\
\hline Cradle Mountain (Site 1) & E1 & BD-2018-05 (HO594860) & S $41^{\circ} 34.753^{\prime}$ E $145^{\circ} 56.199^{\prime}$ & 884 \\
\hline Cradle Mountain (Site 1) & $\mathrm{F} 1$ & BD-2018-06 (HO594861) & S $41^{\circ} 34.751^{\prime}$ E $145^{\circ} 56.186^{\prime}$ & 889 \\
\hline Cradle Mountain (Site 1) & G1 & BD-2018-07 (HO594861) & S $41^{\circ} 34.748^{\prime}$ E $145^{\circ} 56.188^{\prime}$ & 889 \\
\hline Cradle Mountain (Site 1) & $\mathrm{H} 1$ & BD-2018-08 (HO594861) & S $41^{\circ} 34.745^{\prime}$ E $145^{\circ} 56.187^{\prime}$ & 891 \\
\hline Cradle Mountain (Site 1) & 11 & BD-2018-09 (HO594861) & S $41^{\circ} 34.743^{\prime}$ E $145^{\circ} 56.192^{\prime}$ & 892 \\
\hline Cradle Mountain (Site 1) & J1 & BD-2018-10 (HO594861) & S $41^{\circ} 34.740^{\prime}$ E $145^{\circ} 56.197^{\prime}$ & 892 \\
\hline Mount Field (Site 2) & A2 & BD-2018-11 (HO5955163) & S $42^{\circ} 41.080^{\prime}$ E $146^{\circ} 35.406^{\prime}$ & 1076 \\
\hline Mount Field (Site 2) & B2 & BD-2018-12 (HO5955163) & $S 42^{\circ} 41.080^{\prime}$ E $146^{\circ} 35.402^{\prime}$ & 1071 \\
\hline Mount Field (Site 2) & $\mathrm{C} 2$ & BD-2018-13 (HO5955163) & S $42^{\circ} 41.079^{\prime}$ E $146^{\circ} 35.398^{\prime}$ & 1069 \\
\hline Mount Field (Site 2) & D2 & BD-2018-14 (HO5955163) & S $42^{\circ} 41.076^{\prime}$ E $146^{\circ} 35.394^{\prime}$ & 1067 \\
\hline Mount Field (Site 2) & E2 & BD-2018-15 (HO5955162) & S $42^{\circ} 40.946^{\prime}$ E $146^{\circ} 35.357^{\prime}$ & 1076 \\
\hline Mount Field (Site 2) & $\mathrm{F} 2$ & BD-2018-16 (HO5955162) & $S 42^{\circ} 40.945^{\prime}$ E $146^{\circ} 35.357^{\prime}$ & 1073 \\
\hline Mount Field (Site 2) & G2 & BD-2018-17 (HO5955162) & S $42^{\circ} 40.905^{\prime}$ E $146^{\circ} 35.358^{\prime}$ & 1079 \\
\hline Mount Field (Site 2) & $\mathrm{H} 2$ & BD-2018-18 (HO5955162) & $S 42^{\circ} 40.875^{\prime}$ E $146^{\circ} 35.352^{\prime}$ & 1083 \\
\hline Mount Field (Site 2) & 12 & BD-2018-19 (HO5955162) & $S 42^{\circ} 40.865^{\prime}$ E $146^{\circ} 35.344^{\prime}$ & 1083 \\
\hline Mount Wellington (Site 3) & A3 & BD-2018-20 (HO5955161) & S $42^{\circ} 53.088^{\prime}$ E $147^{\circ} 13.302^{\prime}$ & 1111 \\
\hline Mount Wellington (Site 3) & B3 & BD-2018-21 (HO5955161) & S $42^{\circ} 53.084^{\prime}$ E $147^{\circ} 13.304^{\prime}$ & 1113 \\
\hline Mount Wellington (Site 3) & C3 & BD-2018-22 (HO5955161) & S $42^{\circ} 53.083^{\prime}$ E $147^{\circ} 13.302^{\prime}$ & 1114 \\
\hline Mount Wellington (Site 3) & D3 & BD-2018-23 (HO5955161) & S $42^{\circ} 53.078^{\prime}$ E $147^{\circ} 13.297^{\prime}$ & 1117 \\
\hline Mount Wellington (Site 3) & E3 & BD-2018-24 (HO5955161) & S $42^{\circ} 53.072^{\prime}$ E $147^{\circ} 13.299^{\prime}$ & 1121 \\
\hline Mount Wellington (Site 3) & F3 & BD-2018-25 (HO5955161) & S $42^{\circ} 53.068^{\prime}$ E $147^{\circ} 13.294^{\prime}$ & 1121 \\
\hline Mount Wellington (Site 3) & G3 & BD-2018-26 (HO5955160) & S $42^{\circ} 53.064^{\prime}$ E $147^{\circ} 13.288^{\prime}$ & 1123 \\
\hline Mount Wellington (Site 3) & $\mathrm{H} 3$ & BD-2018-27 (HO5955160) & S $42^{\circ} 53.060^{\prime}$ E $147^{\circ} 13.288^{\prime}$ & 1127 \\
\hline Mount Wellington (Site 3) & 13 & BD-2018-28 (HO5955160) & S $42^{\circ} 53.042^{\prime}$ E $147^{\circ} 13.272^{\prime}$ & 1135 \\
\hline Mount Wellington (Site 3) & J3 & BD-2018-29 (HO5955160) & S $42^{\circ} 53.049^{\prime}$ E $147^{\circ} 13.258^{\prime}$ & 1134 \\
\hline Mount Wellington (Site 3) & K3 & BD-2018-30 (HO5955160) & $S 42^{\circ} 53.060^{\prime}$ E $147^{\circ} 13.260^{\prime}$ & 1128 \\
\hline
\end{tabular}


Table S1B. T. lanceolata population sites \& voucher details (continued).

\begin{tabular}{|c|c|c|c|c|}
\hline Population Site & Sample & Specimen ID (Population Voucher Plate \#) & GPS Location & Altitude (m) \\
\hline Mount Wellington (Site 4) & A4 & BD-2018-31 (HO5955159) & S $42^{\circ} 54.313^{\prime}$ E $147^{\circ} 15.027^{\prime}$ & 627 \\
\hline Mount Wellington (Site 4) & B4 & BD-2018-32 (HO5955159) & S $42^{\circ} 54.358^{\prime}$ E $147^{\circ} 14.968^{\prime}$ & 627 \\
\hline Mount Wellington (Site 4) & $\mathrm{C} 4$ & BD-2018-33 (HO5955159) & S $42^{\circ} 54.361^{\prime}$ E $147^{\circ} 14.964^{\prime}$ & 625 \\
\hline Mount Wellington (Site 4) & D4 & BD-2018-34 (HO5955159) & S $42^{\circ} 54.368^{\prime}$ E $147^{\circ} 14.951^{\prime}$ & 627 \\
\hline Mount Wellington (Site 4) & E4 & BD-2018-35 (HO5955158) & S $42^{\circ} 54.369^{\prime}$ E $147^{\circ} 14.944^{\prime}$ & 631 \\
\hline Mount Wellington (Site 4) & $\mathrm{F} 4$ & BD-2018-36 (HO5955158) & S $42^{\circ} 54.379^{\prime}$ E $147^{\circ} 14.930^{\prime}$ & 636 \\
\hline Mount Wellington (Site 4) & G4 & BD-2018-37 (HO5955158) & S $42^{\circ} 54.378^{\prime}$ E $147^{\circ} 14.931^{\prime}$ & 638 \\
\hline Mount Wellington (Site 4) & $\mathrm{H} 4$ & BD-2018-38 (HO5955157) & S $42^{\circ} 54.384^{\prime}$ E $147^{\circ} 14.934^{\prime}$ & 633 \\
\hline Mount Wellington (Site 4) & 14 & BD-2018-39 (HO5955157) & S $42^{\circ} 54.386^{\prime}$ E $147^{\circ} 14.933^{\prime}$ & 634 \\
\hline Mount Wellington (Site 4) & J4 & BD-2018-40 (HO5955157) & S $42^{\circ} 54.386^{\prime}$ E $147^{\circ} 14.936^{\prime}$ & 632 \\
\hline Bronzewing "Population A" & A & BD-2018-41 (HO5955156) & S 41.285039 E $147.214871^{\circ}$ & $\sim 250$ \\
\hline Bronzewing "Population B" & $B$ & BD-2018-42 (HO5955156) & $S 41.285039^{\circ}$ E $147.214871^{\circ}$ & $\sim 250$ \\
\hline
\end{tabular}

\section{Yield Data: Drimane Sesquiterpenoids (from Heptane Extracts)}

Table S2A. Yields of drimane sesquiterpenoids from sampled $T$. lanceolata populations.

\begin{tabular}{|c|c|c|c|c|c|}
\hline Population Site & Sample & $\begin{array}{l}\text { Altitude } \\
(\mathrm{m})\end{array}$ & $\begin{array}{l}\text { Polygodial (1) } \\
(\% \mathrm{w} / \mathrm{w})\end{array}$ & $\begin{array}{c}1 \beta \text {-acetoxy-9-deoxyisomuzigadial (6) } \\
(\% \mathrm{w} / \mathrm{w})\end{array}$ & $\begin{array}{c}3 \beta \text {-acetoxydrimenin }(11) \\
(\% \mathrm{w} / \mathrm{w})\end{array}$ \\
\hline Mount Wellington (Site 4) & A4 & 627 & 1.13 & - & - \\
\hline Mount Wellington (Site 4) & B4 & 627 & 1.80 & - & - \\
\hline Mount Wellington (Site 4) & $\mathrm{C} 4$ & 625 & 0.77 & - & - \\
\hline Mount Wellington (Site 4) & D4 & 627 & 0.37 & - & - \\
\hline Mount Wellington (Site 4) & E4 & 631 & 1.23 & - & - \\
\hline Mount Wellington (Site 4) & $\mathrm{F} 4$ & 636 & 0.48 & - & - \\
\hline Mount Wellington (Site 4) & G4 & 638 & 0.79 & - & - \\
\hline Mount Wellington (Site 4) & $\mathrm{H} 4$ & 633 & 1.03 & - & - \\
\hline Mount Wellington (Site 4) & 14 & 634 & 0.93 & - & - \\
\hline Mount Wellington (Site 4) & $\mathrm{J} 4$ & 632 & 0.86 & - & - \\
\hline Cradle Mountain (Site 1) & A1 & 888 & 0.22 & - & - \\
\hline Cradle Mountain (Site 1) & B1 & 886 & 0.64 & - & - \\
\hline Cradle Mountain (Site 1) & $\mathrm{C} 1$ & 887 & 0.19 & - & - \\
\hline Cradle Mountain (Site 1) & D1 & 884 & 0.61 & - & - \\
\hline Cradle Mountain (Site 1) & E1 & 884 & 0.51 & - & - \\
\hline
\end{tabular}


Table S2B. Yields of drimane sesquiterpenoids from sampled $T$. lanceolata populations (continued).

\begin{tabular}{|c|c|c|c|c|c|}
\hline Population Site & Sample & $\begin{array}{l}\text { Altitude } \\
(\mathrm{m})\end{array}$ & $\begin{array}{l}\text { Polygodial (1) } \\
(\% \mathrm{w} / \mathrm{w})\end{array}$ & $\begin{array}{c}1 \beta \text {-acetoxy-9-deoxyisomuzigadial (6) } \\
(\% \mathrm{w} / \mathrm{w})\end{array}$ & $\begin{array}{l}3 \beta \text {-acetoxydrimenin (11) } \\
(\% \mathrm{w} / \mathrm{w})\end{array}$ \\
\hline Cradle Mountain (Site 1) & F1 & 889 & 0.28 & - & - \\
\hline Cradle Mountain (Site 1) & G1 & 889 & 0.14 & - & - \\
\hline Cradle Mountain (Site 1) & $\mathrm{H} 1$ & 891 & 0.15 & - & - \\
\hline Cradle Mountain (Site 1) & 11 & 892 & 0.21 & - & - \\
\hline Cradle Mountain (Site 1) & J1 & 892 & 0.25 & - & - \\
\hline Mount Field (Site 2) & A2 & 1076 & - & 0.17 & $<0.1$ (trace) \\
\hline Mount Field (Site 2) & B2 & 1071 & - & 0.33 & $<0.1$ (trace) \\
\hline Mount Field (Site 2) & $\mathrm{C} 2$ & 1069 & - & 0.07 & $<0.1$ (trace) \\
\hline Mount Field (Site 2) & $\mathrm{D} 2$ & 1067 & - & 0.13 & $<0.1$ (trace) \\
\hline Mount Field (Site 2) & E2 & 1076 & - & 0.15 & $<0.1$ (trace) \\
\hline Mount Field (Site 2) & $\mathrm{F} 2$ & 1073 & - & 0.22 & $<0.1$ (trace) \\
\hline Mount Field (Site 2) & G2 & 1079 & - & 0.05 & $<0.1$ (trace) \\
\hline Mount Field (Site 2) & $\mathrm{H} 2$ & 1083 & - & 0.09 & $<0.1$ (trace) \\
\hline Mount Field (Site 2) & 12 & 1083 & - & 0.14 & $<0.1$ (trace) \\
\hline Mount Wellington (Site 3) & A3 & 1111 & - & 0.09 & $<0.1$ (trace) \\
\hline Mount Wellington (Site 3) & B3 & 1113 & - & 0.07 & $<0.1$ (trace) \\
\hline Mount Wellington (Site 3) & C3 & 1114 & - & 0.05 & $<0.1$ (trace) \\
\hline Mount Wellington (Site 3) & D3 & 1117 & - & 0.07 & $<0.1$ (trace) \\
\hline Mount Wellington (Site 3) & E3 & 1121 & - & 0.03 & $<0.1$ (trace) \\
\hline Mount Wellington (Site 3) & F3 & 1121 & - & 0.05 & $<0.1$ (trace) \\
\hline Mount Wellington (Site 3) & G3 & 1123 & - & 0.02 & $<0.1$ (trace) \\
\hline Mount Wellington (Site 3) & $\mathrm{H} 3$ & 1127 & - & 0.06 & $<0.1$ (trace) \\
\hline Mount Wellington (Site 3) & 13 & 1135 & - & 0.05 & $<0.1$ (trace) \\
\hline Mount Wellington (Site 3) & $\mathrm{J} 3$ & 1134 & - & 0.06 & $<0.1$ (trace) \\
\hline Mount Wellington (Site 3) & K3 & 1128 & - & 0.05 & $<0.1$ (trace) \\
\hline $\begin{array}{c}\text { Bronzewing "A" } \\
\text { (Commercial) }\end{array}$ & A & N/A & 1.05 & - & - \\
\hline $\begin{array}{c}\text { Bronzewing "B" } \\
\text { (Commercial) }\end{array}$ & B & $N / A$ & 0.94 & - & - \\
\hline $\begin{array}{l}\text { Diemen Pepper } \\
\text { (Commercial) }\end{array}$ & C & $\mathrm{N} / \mathrm{A}$ & 0.47 & - & - \\
\hline
\end{tabular}




\section{X-Ray Crystallographic Data}

Table S3. Crystallographic Data Parameters

\begin{tabular}{|c|c|c|}
\hline Compound & 11 & 23 \\
\hline empirical formula & $2\left(\mathrm{C}_{16} \mathrm{H}_{18} \mathrm{O}_{9}\right) \mathrm{H}_{2} \mathrm{O}$ & $\mathrm{C}_{17} \mathrm{H}_{24} \mathrm{O}_{4}$ \\
\hline fw (g mol-1) & 726.62 & 292.36 \\
\hline crystal system & orthorhombic & orthorhombic \\
\hline space group & $P 2{ }_{1}{ }_{1} 2{ }_{1}$ & $P 2{ }_{12} 2_{1}$ \\
\hline$a, \AA$ & $7.7620(16)$ & $6.3361(4)$ \\
\hline$b, \AA$ & $11.021(2)$ & $8.6846(4)$ \\
\hline$c, \AA$ & $36.648(7)$ & 27.7189(14) \\
\hline$V, \AA^{3}$ & $3135.1(11)$ & $1525.27(14)$ \\
\hline$D_{\text {calcd }},\left(\mathrm{g} \mathrm{cm}^{-3}\right)$ & 1.539 & 1.273 \\
\hline cryst size, $\mathrm{mm}$ & $0.05 \times 0.01 \times 0.01$ & $0.3 \times 0.25 \times 0.05$ \\
\hline$\mu, \mathrm{mm}^{-1}$ & 0.130 & 0.72 \\
\hline no. unique reflns & 9908 & 3269 \\
\hline no. reflns obs. $(I>2 \sigma(I))$ & 6856 & 3117 \\
\hline$R_{\text {int }}$ & 0.106 & 0.043 \\
\hline temperature, $\mathrm{K}$ & 100 & 150 \\
\hline Final $R_{1}, w R_{2}$ & $0.056,0.150$ & $0.029,0.076$ \\
\hline Flack parameter & - & $0.07(7)$ \\
\hline Hooft parameter & $-0.6(4)$ & $0.09(7)$ \\
\hline P2 (true) & 0.996 & 1.000 \\
\hline P3 (true) & 0.896 & 1.000 \\
\hline P3 (rac-twin) & 0.101 & $0.1 \times 10^{-9}$ \\
\hline P3 (false) & 0.003 & $0.2 \times 10^{-47}$ \\
\hline
\end{tabular}




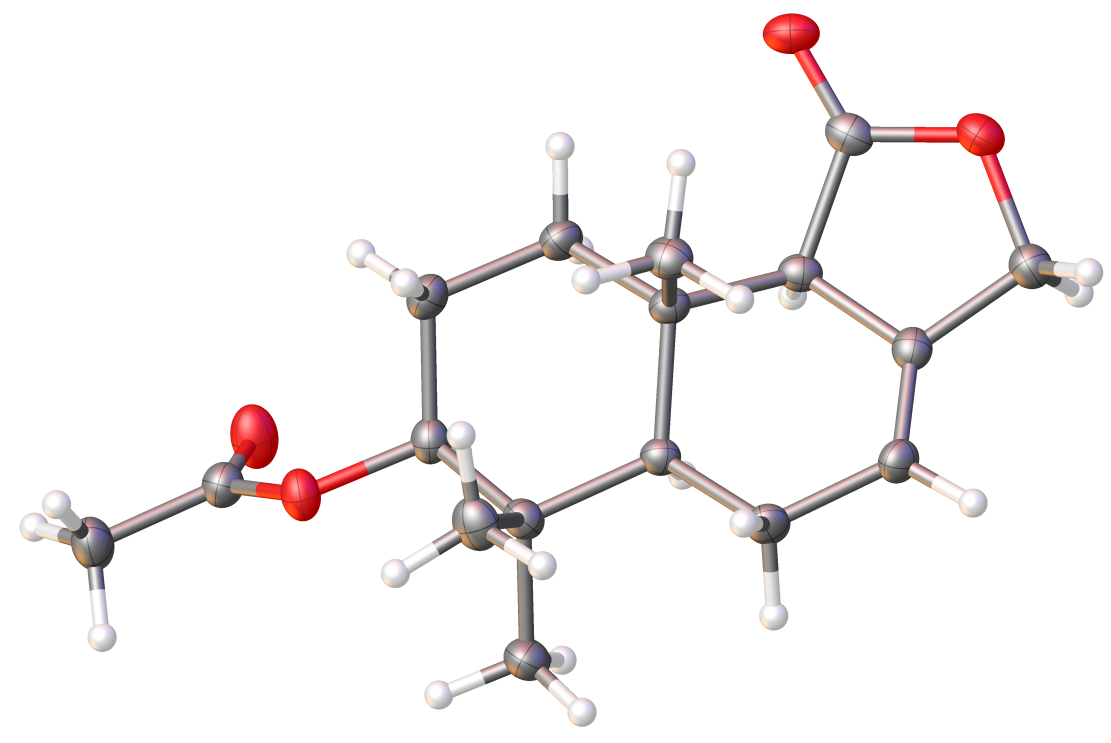

Figure S1. Molecular representation of $3 \beta$-acetoxydrimenin (11). Ellipsoids are shown at $50 \%$ probability.

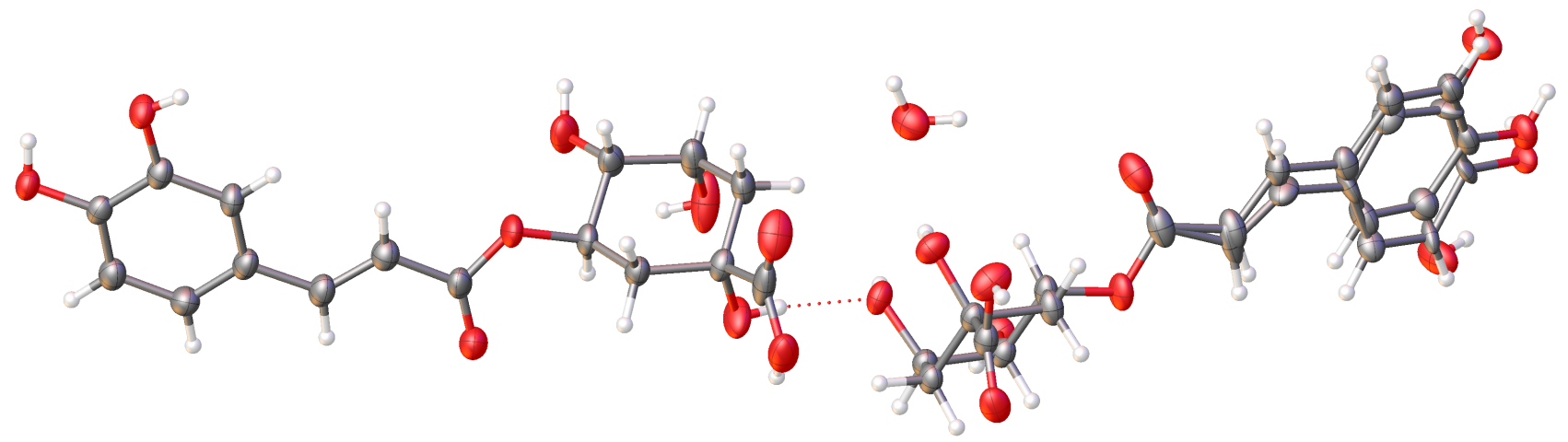

Figure S2. Molecular representation of chlorogenic acid ester (23). Ellipsoids are shown at $50 \%$ probability. 

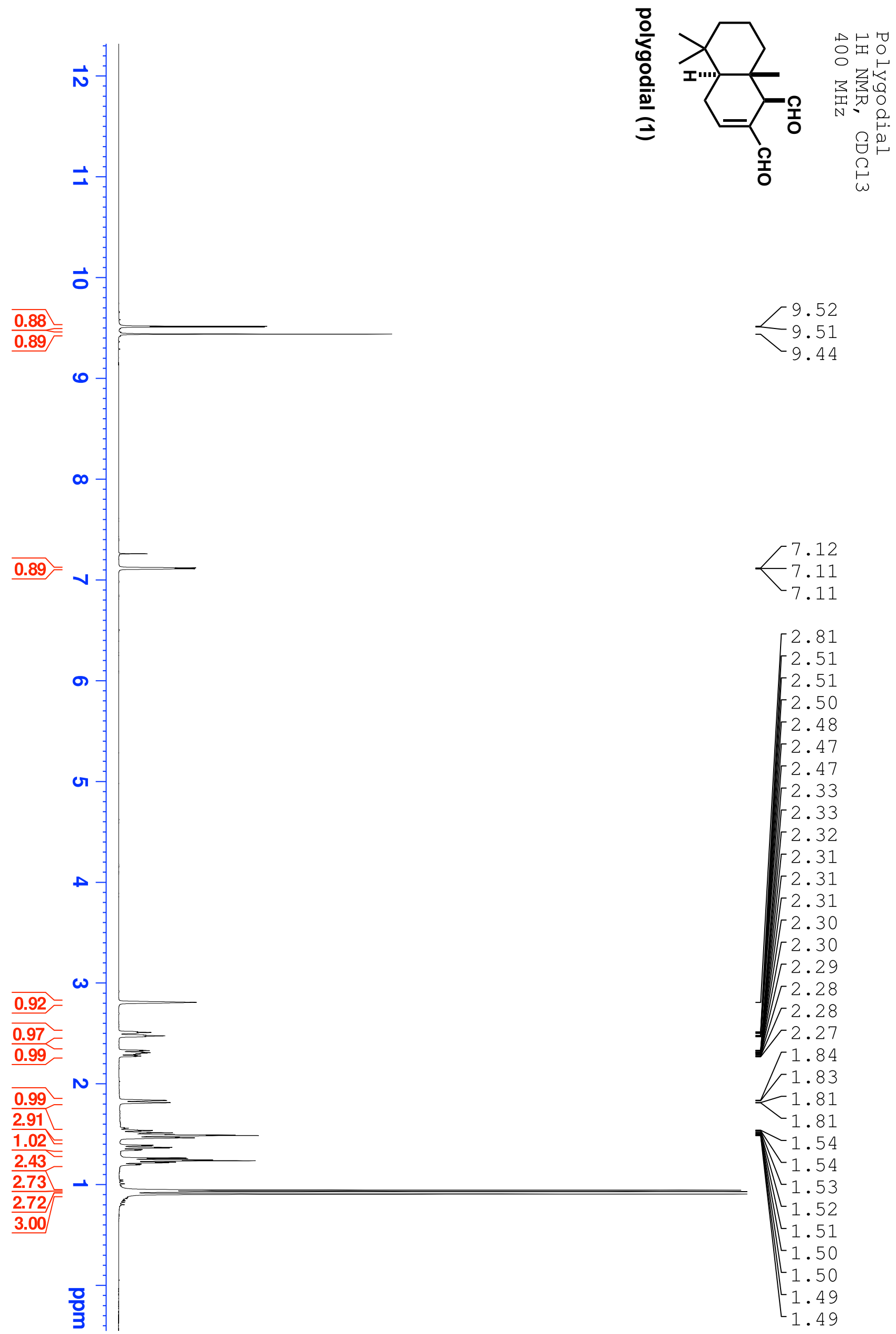

$<\begin{aligned} & 7.12 \\ & 7.11 \\ & 7.11\end{aligned}$

2.81

2.51

$-2.51$

2.50

2.48

2.47

2.47

2.33

2.33

2.32

2.31

2.31

2.31

2.30

[2.30

[2.29

2.28

2.28

2.27

1.84

1.83

$-1.81$

1.81

1.54

$-1.54$

1.53

1.52

1.51

1.50

1.50

1.49

1.49 

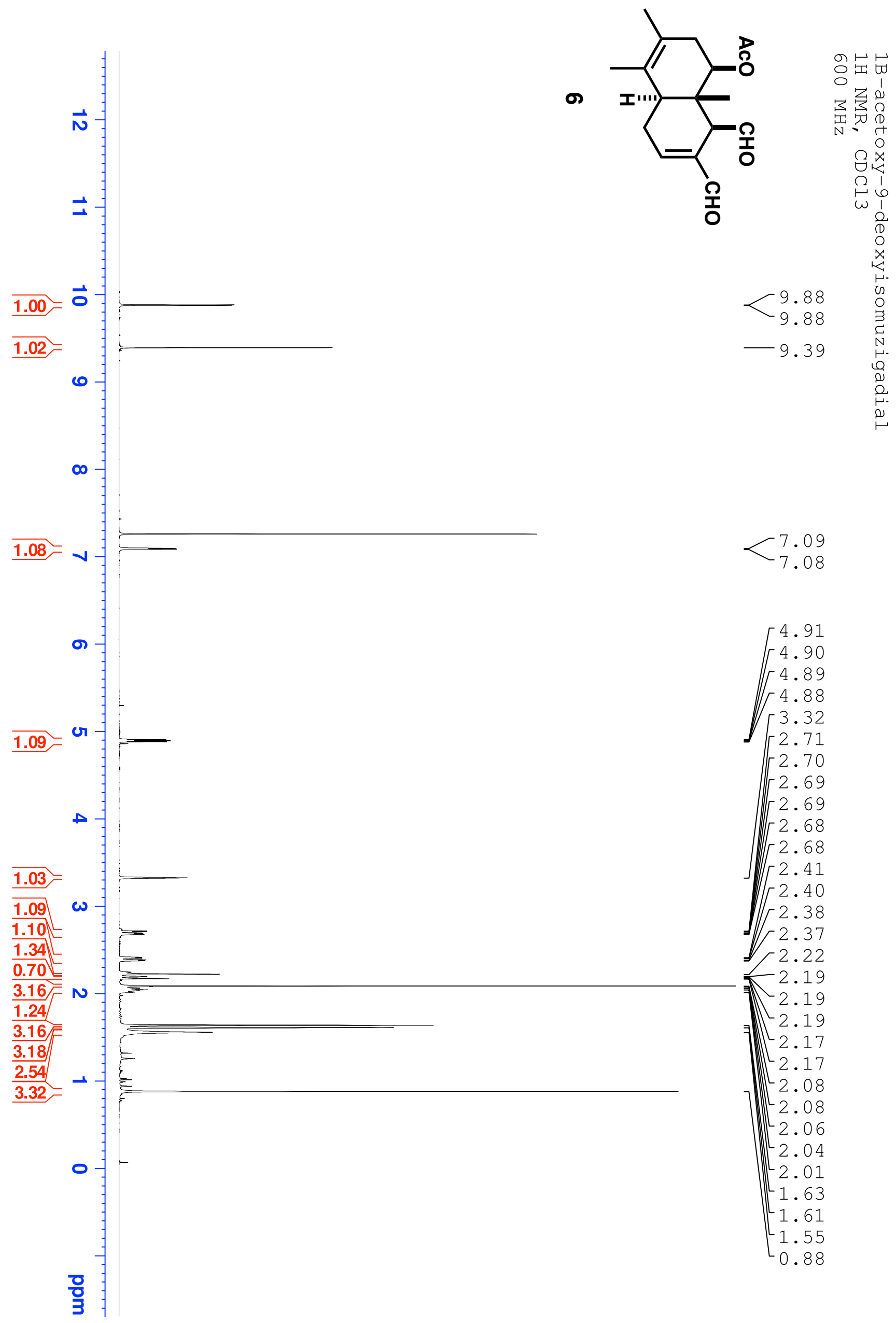

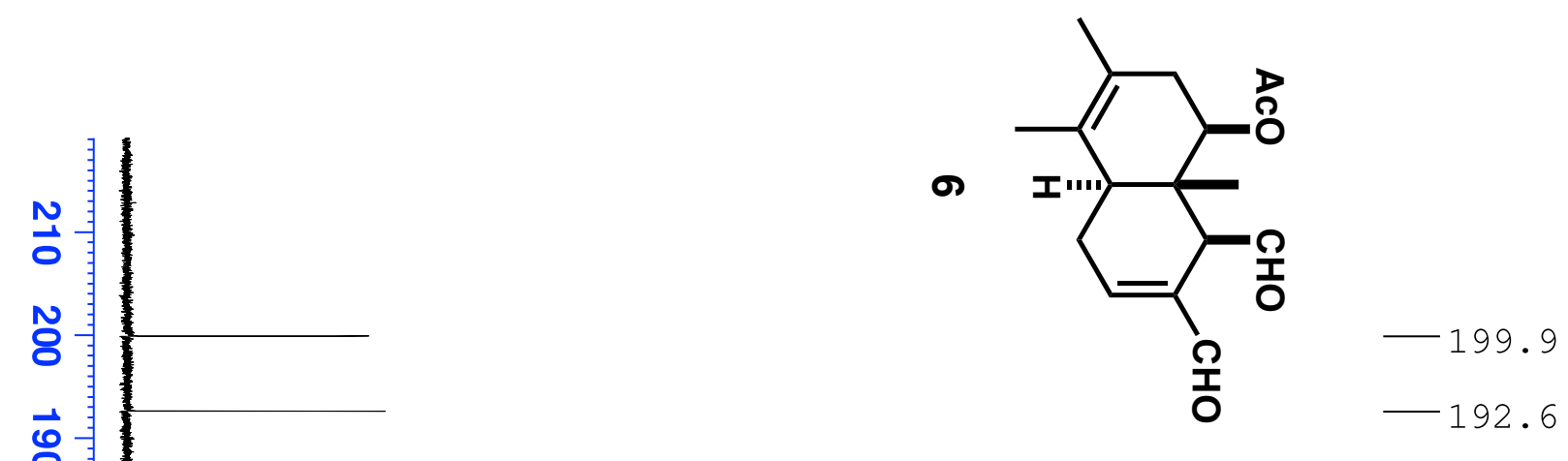

ज $\omega$

○ I

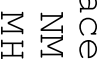

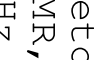

$-199.9$ 离

$-192.6 \Omega 6$

$-170.4$

$-152.2$

$-140.8$

125.1
-124.3

$\overrightarrow{\mathrm{N}}$

$\overrightarrow{0}$

$\overrightarrow{8}$

0

\&

ठै

8

잉

o

జ

ก

$-26.6$

$-21.5$

$-19.0$

$-15.4$

$\overrightarrow{0}$

$-9.6$

믈 


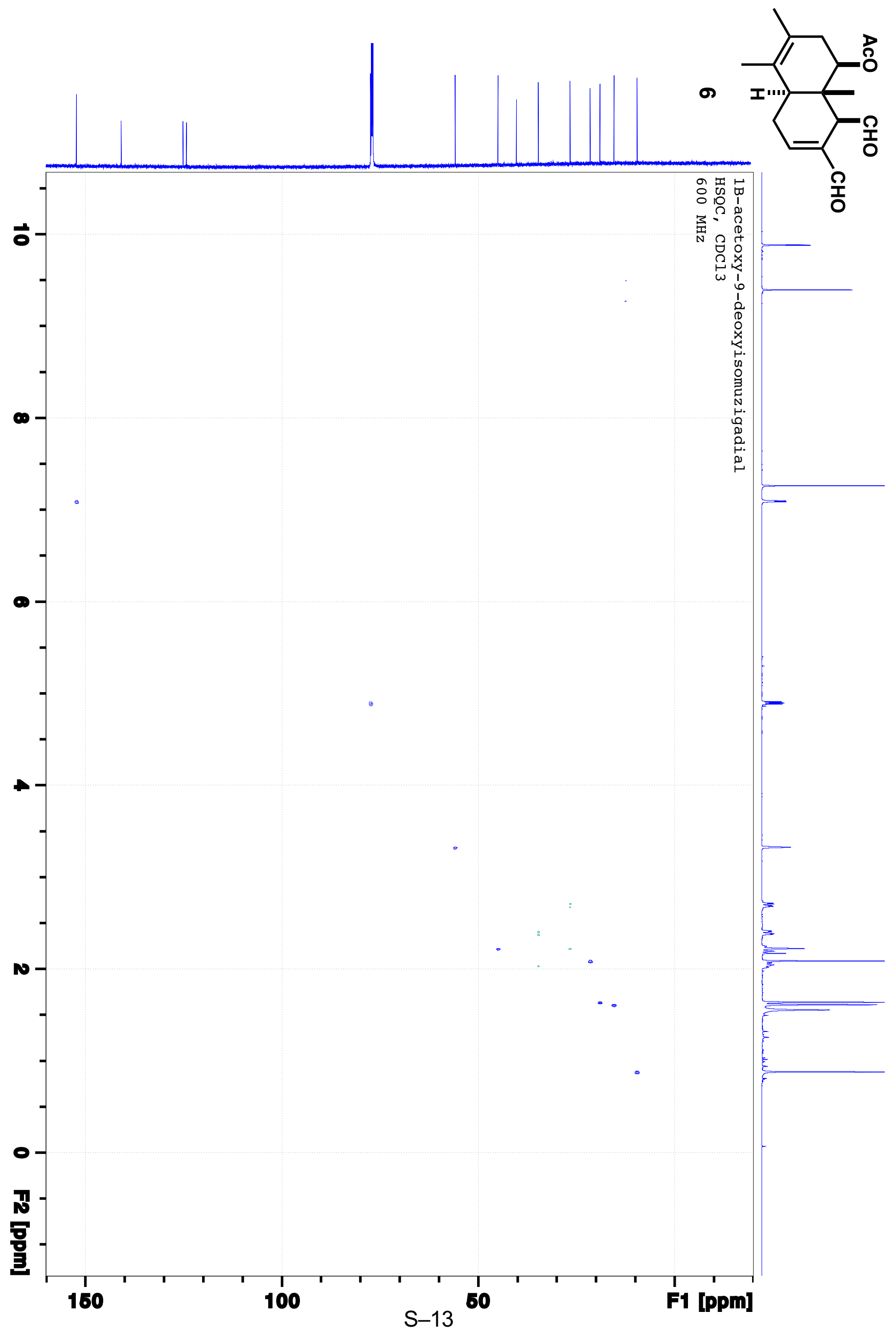




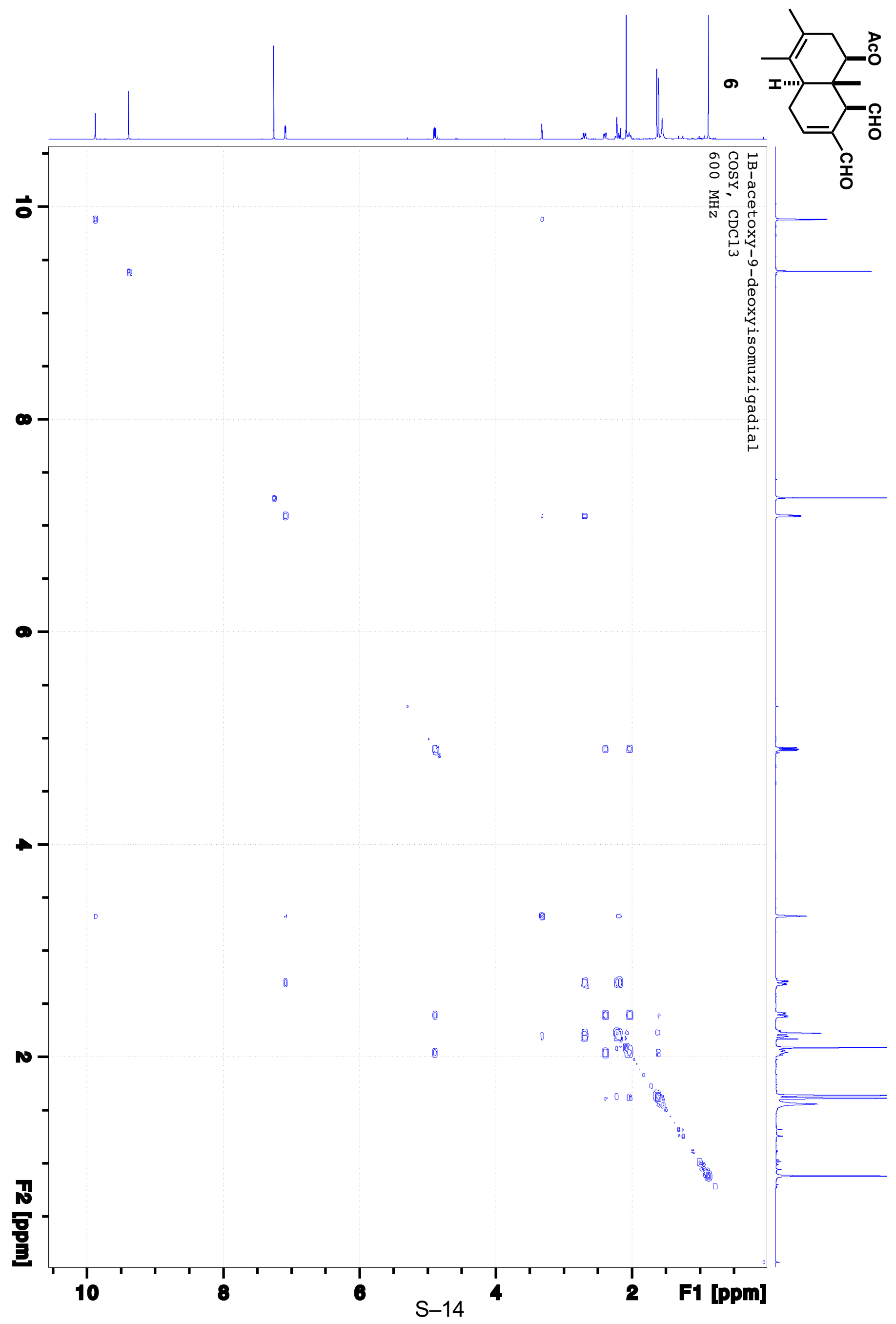




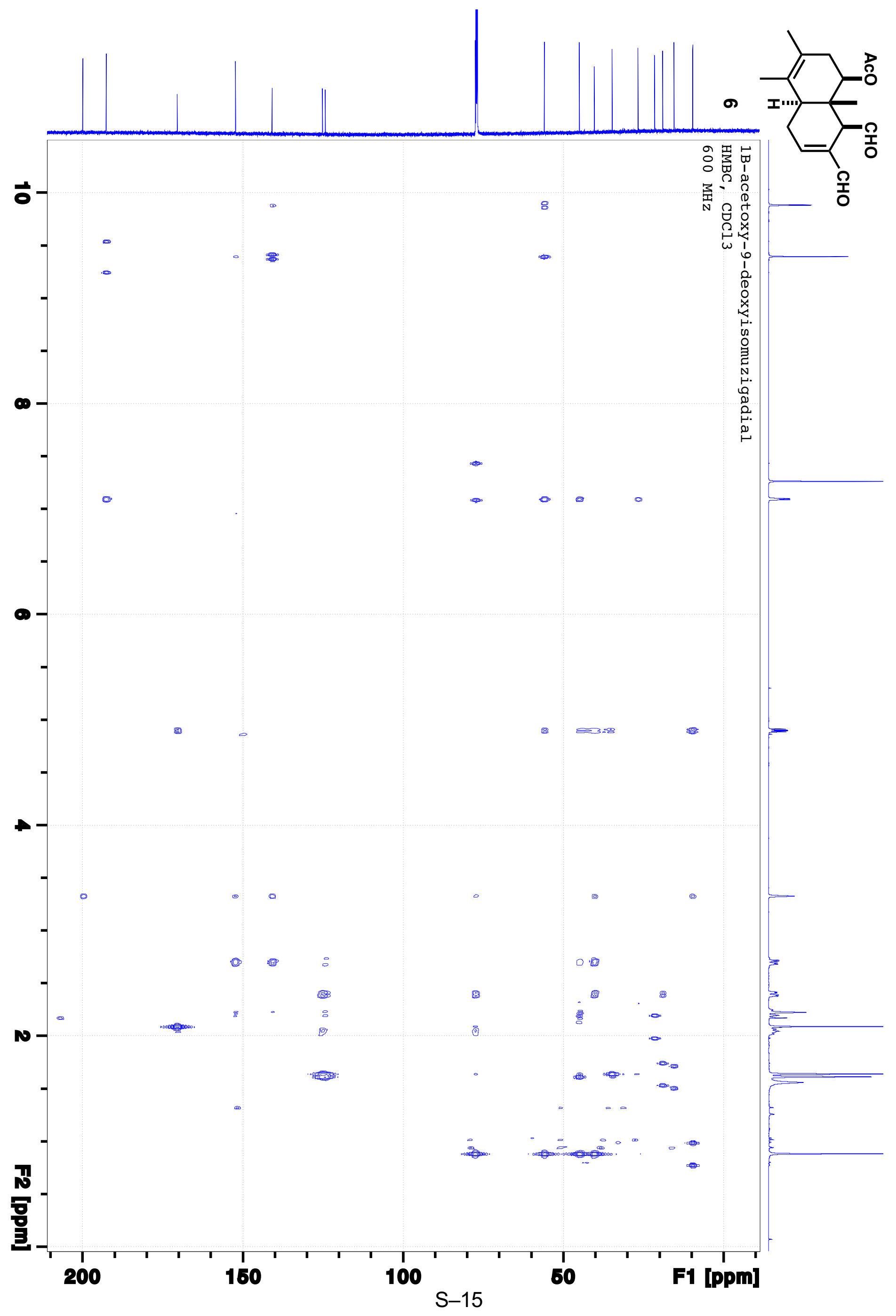



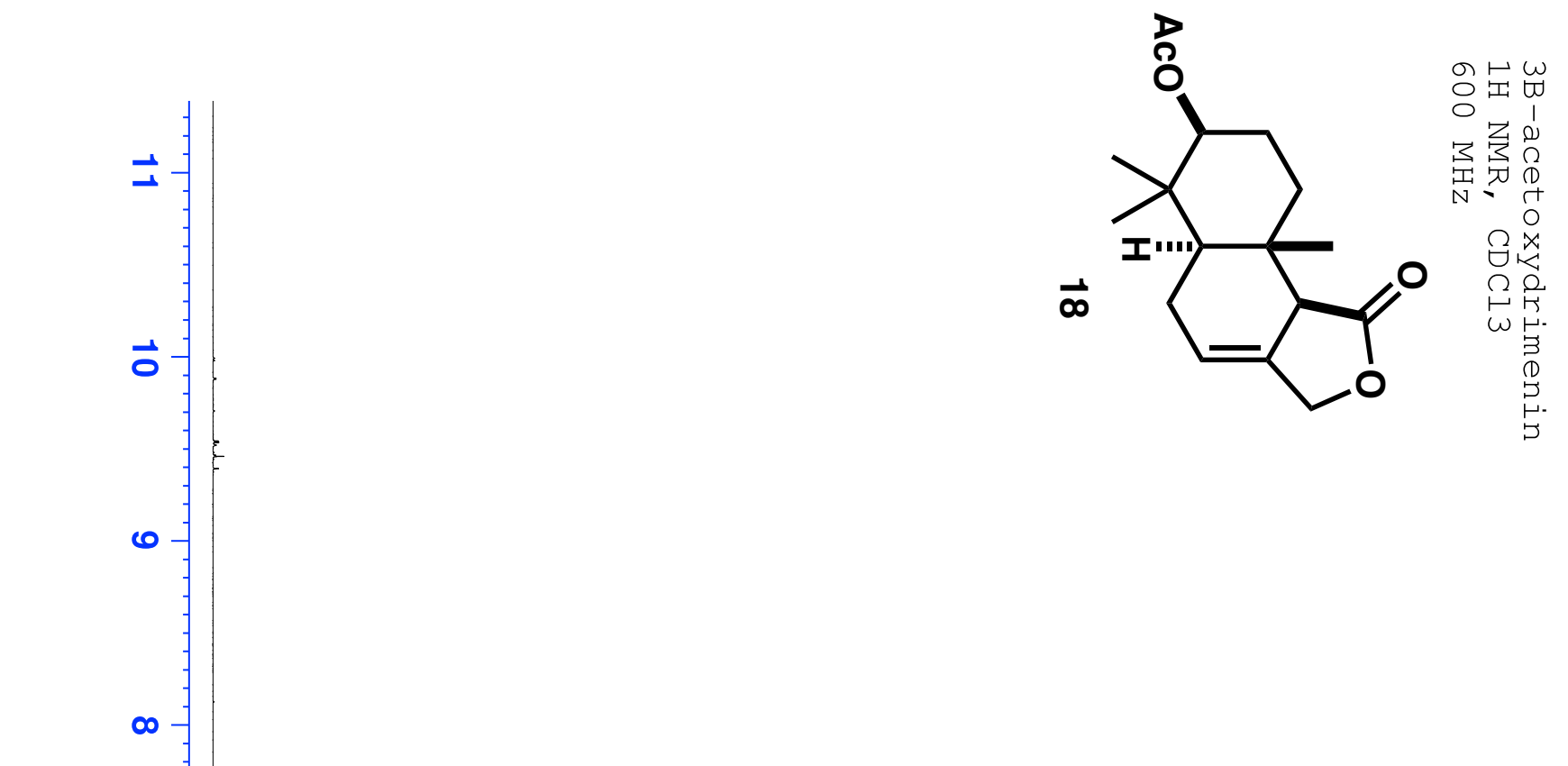

5.75

$-4.71$ 4.71 4.69 4.69 4.69 4.68 4.67 4.65 4.59 $[4.58$ 4.57 4.56 2.77

2.23 [2.23 2.22 2.21 - 2.20

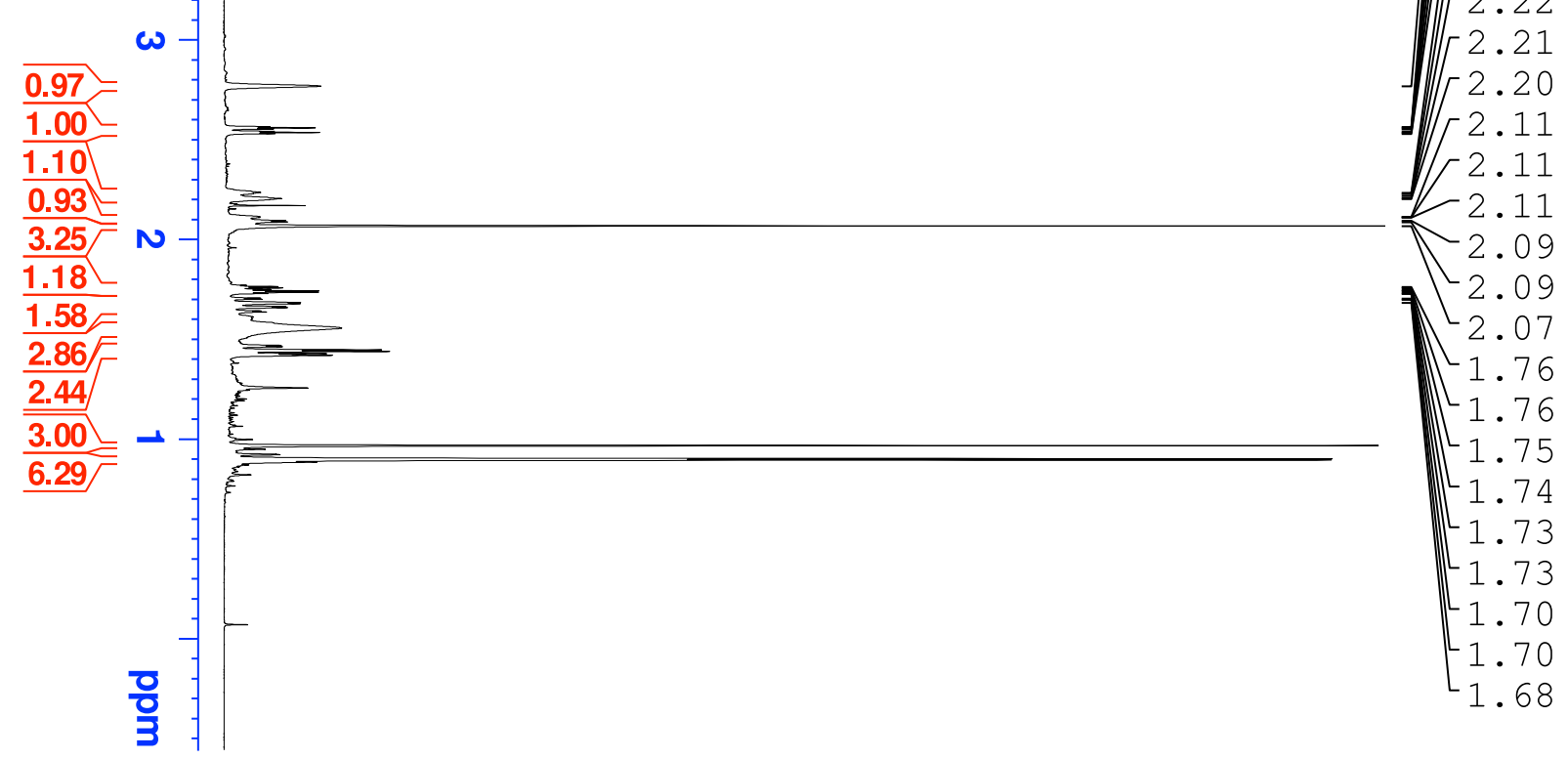



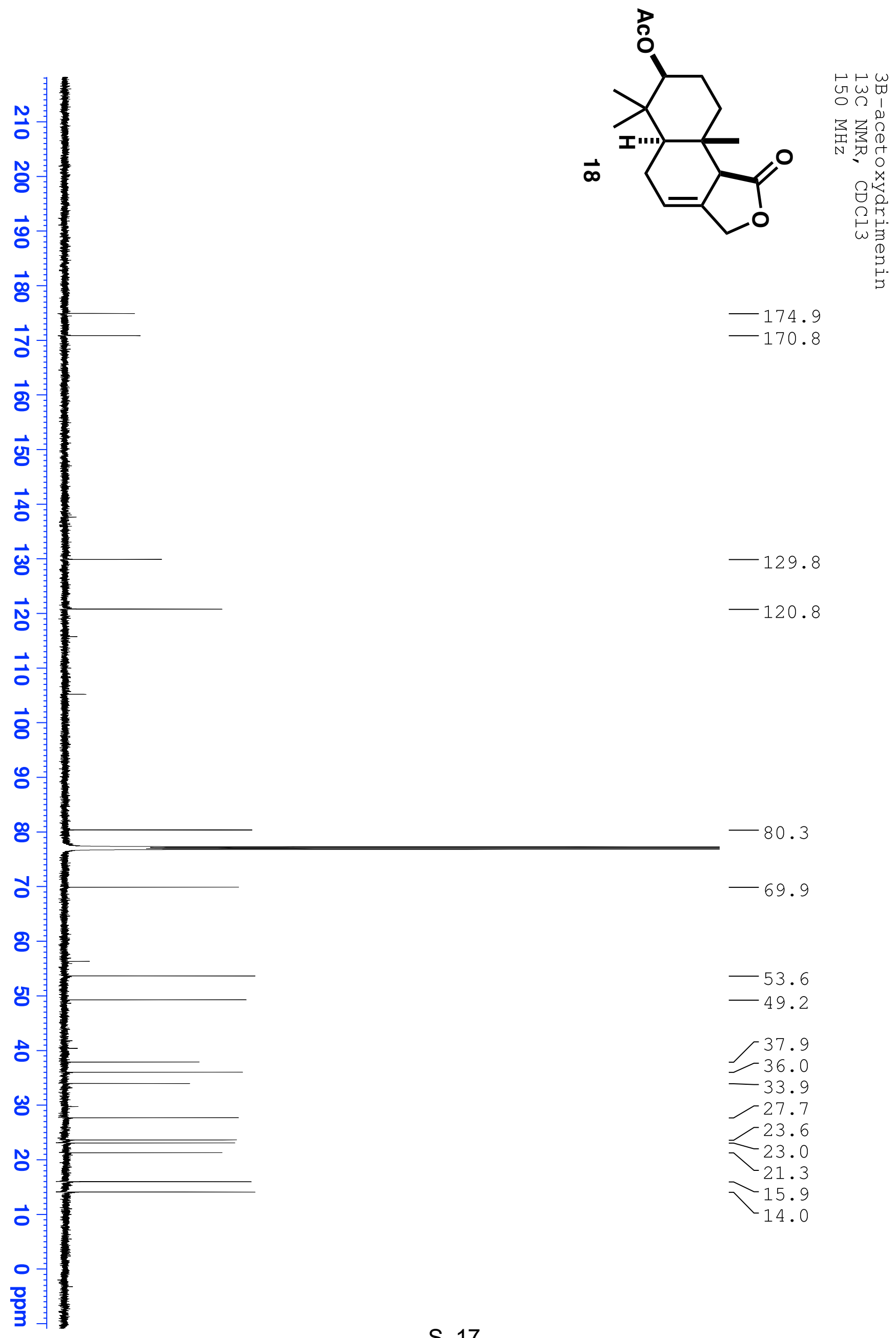

$-129.8$

$-120.8$

$-80.3$

$-69.9$

$-53.6$

$-49.2$

r 37.9

$-36.0$

$-33.9$

$-23.6$

$-23.0$

21.3

$-15.9$

14.0 


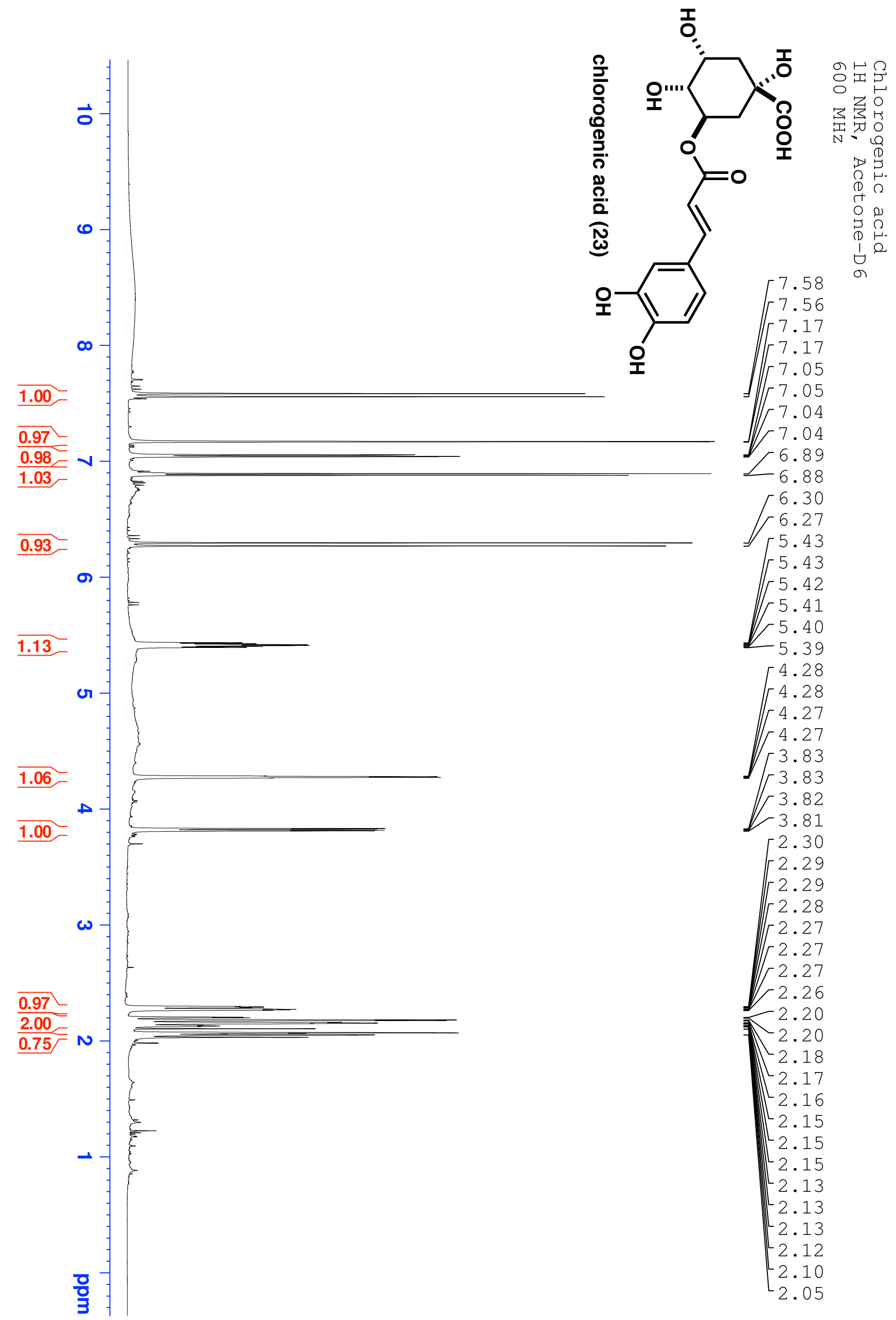




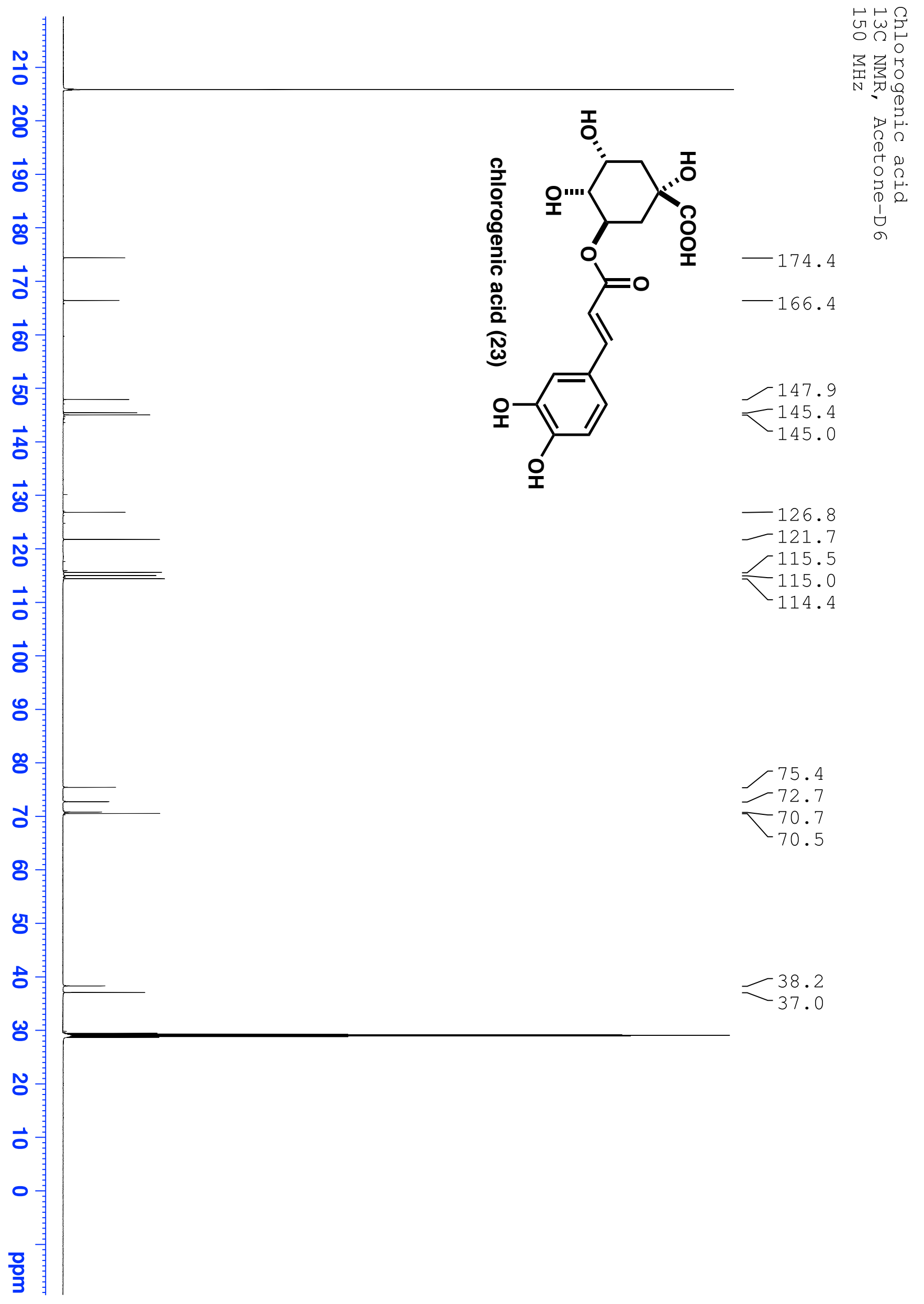



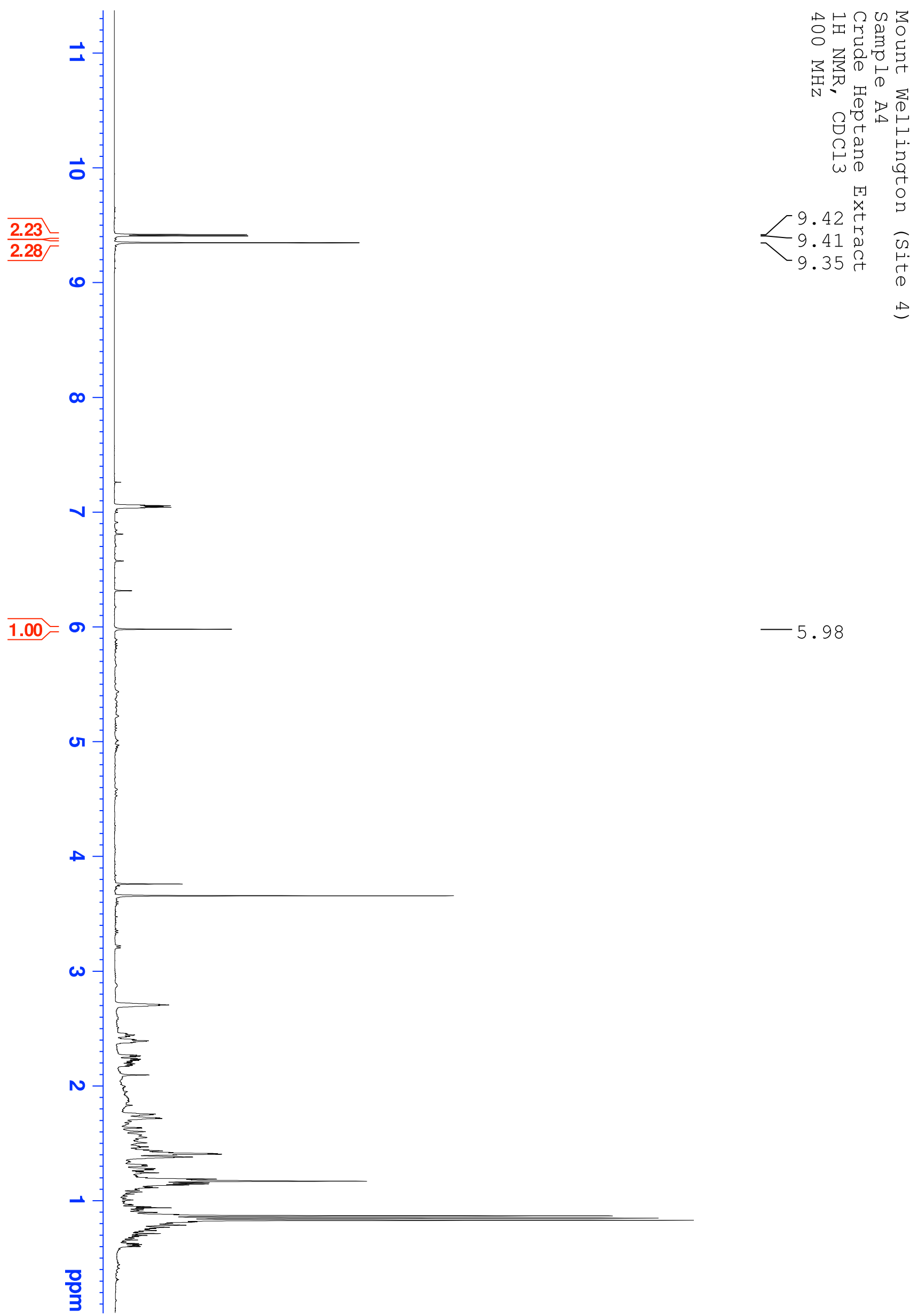

$-5.98$ 


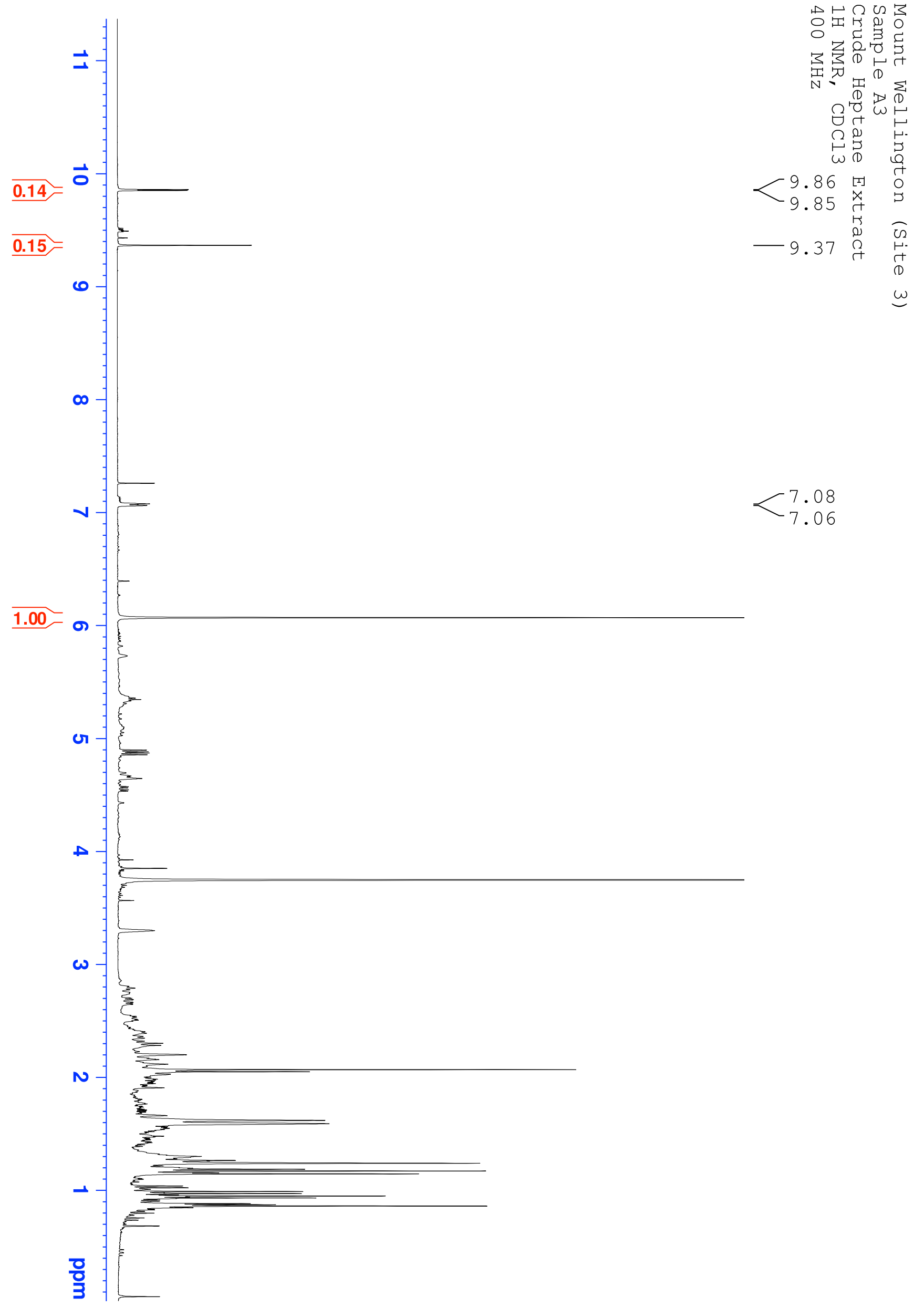




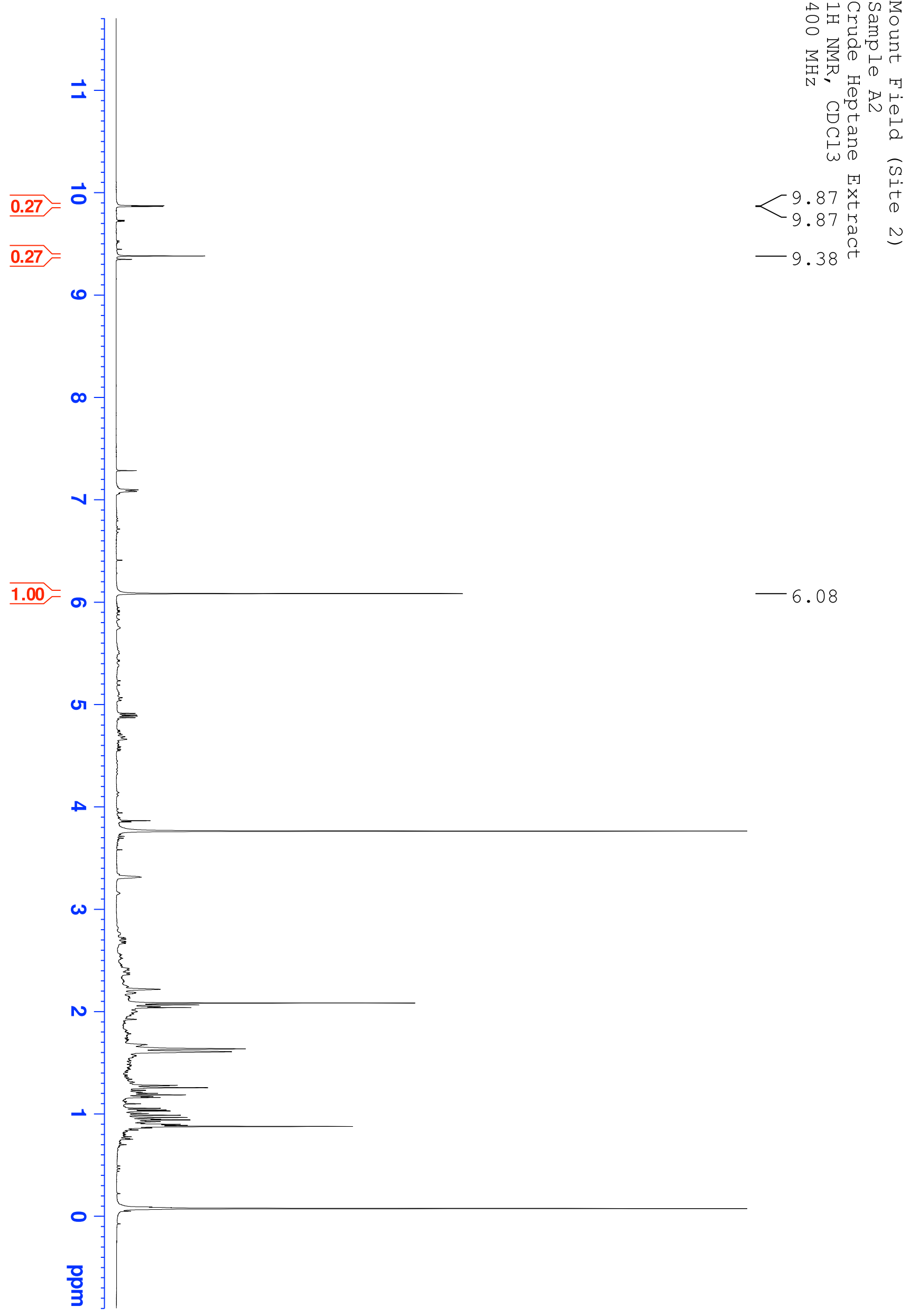




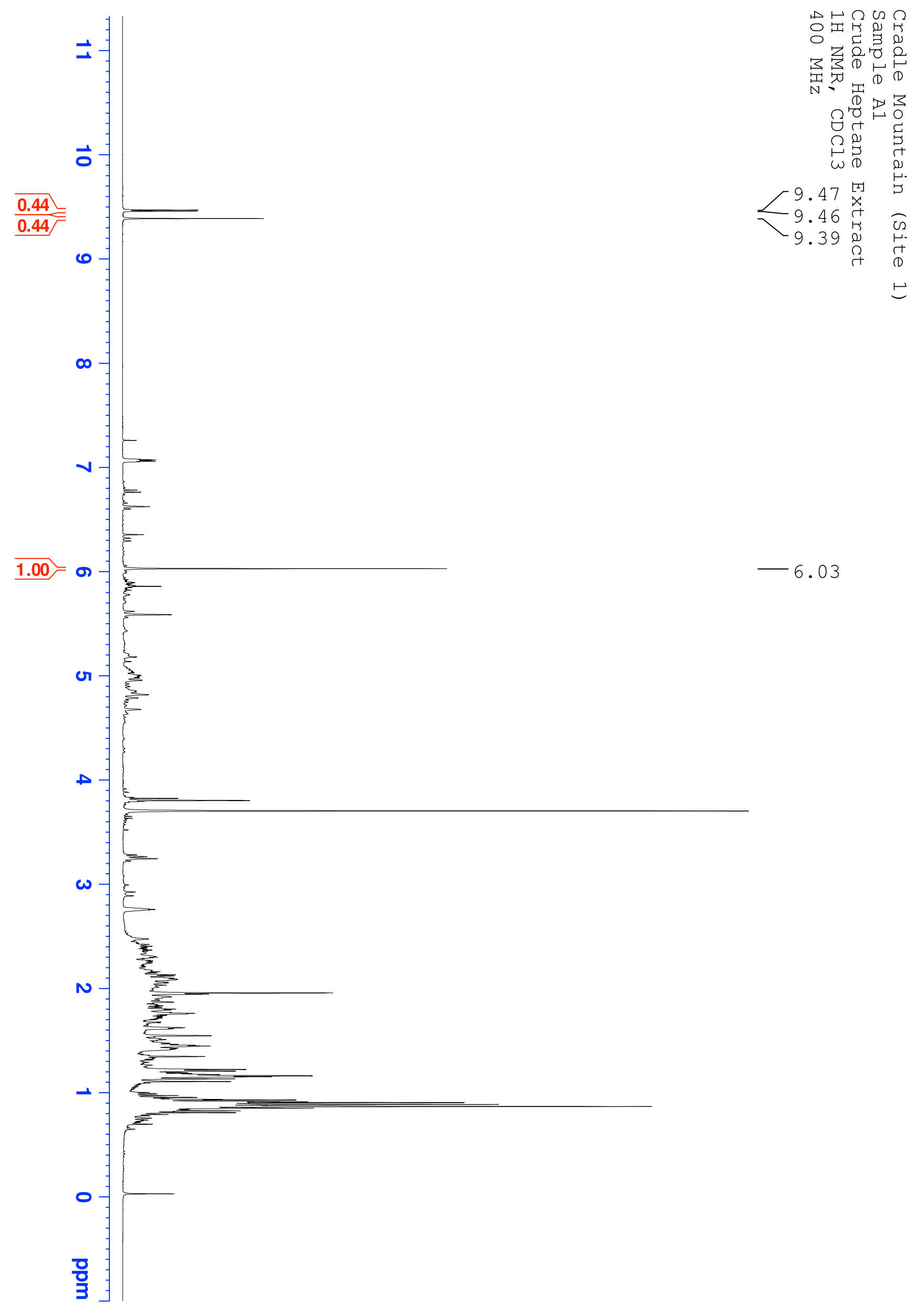




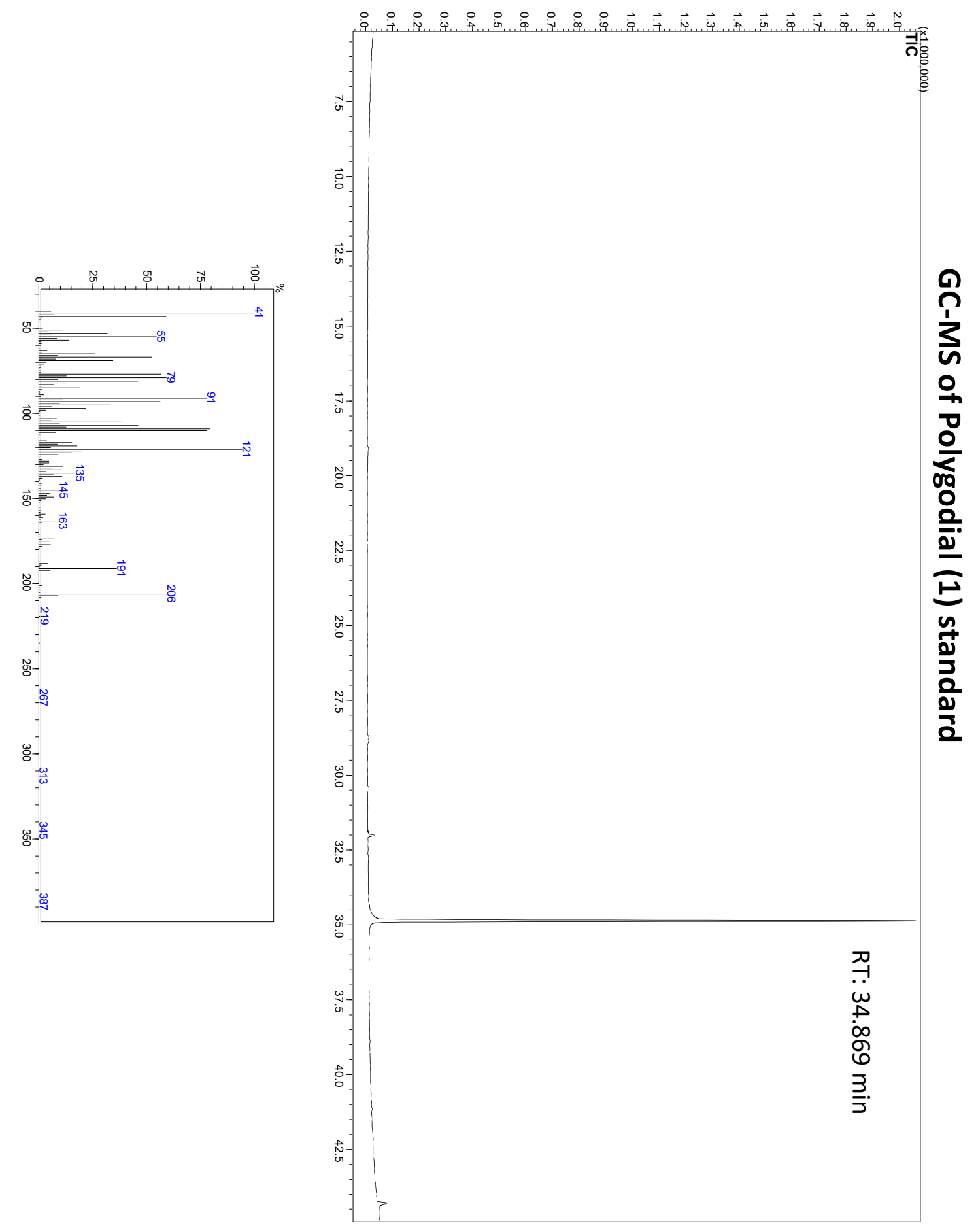




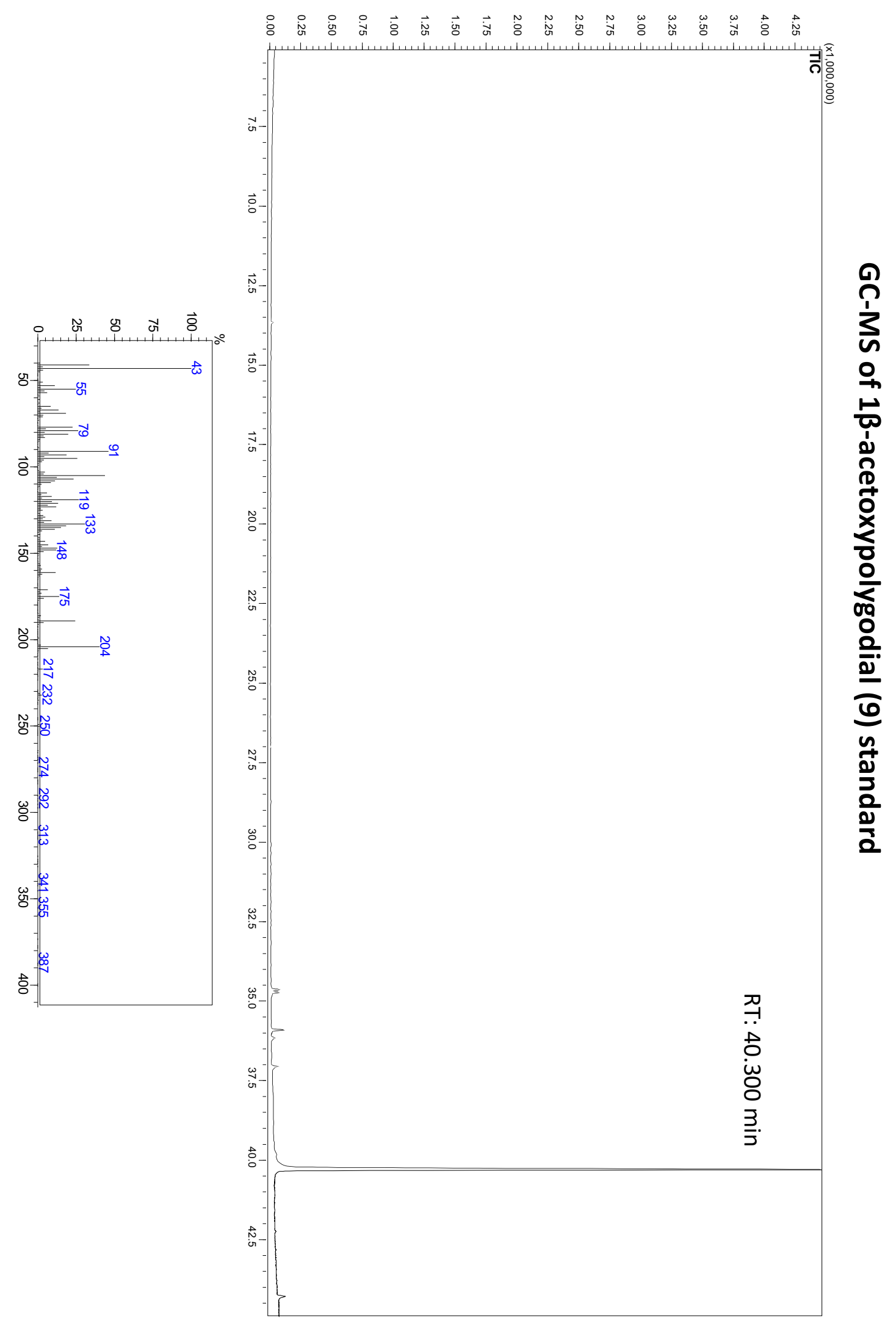




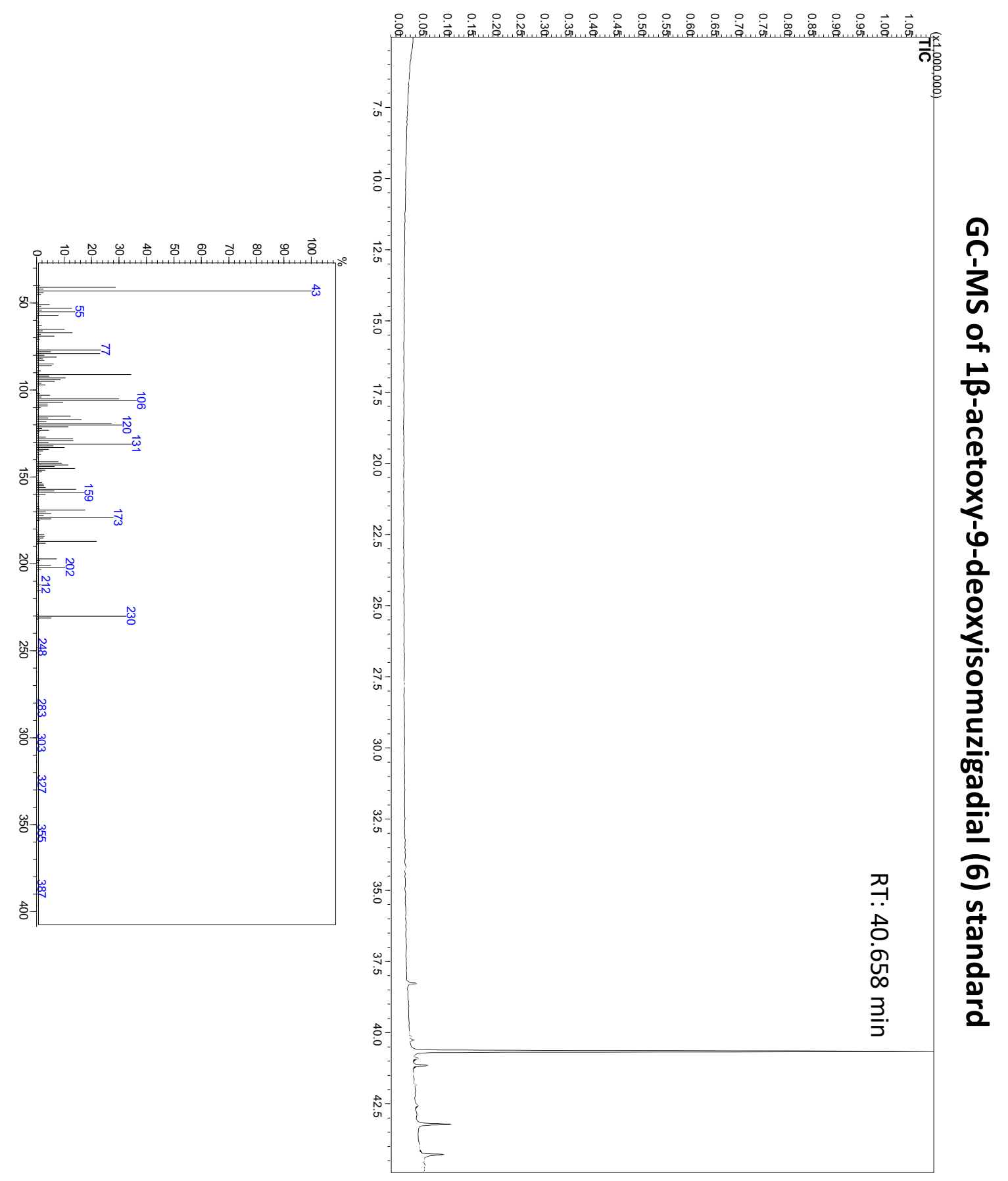




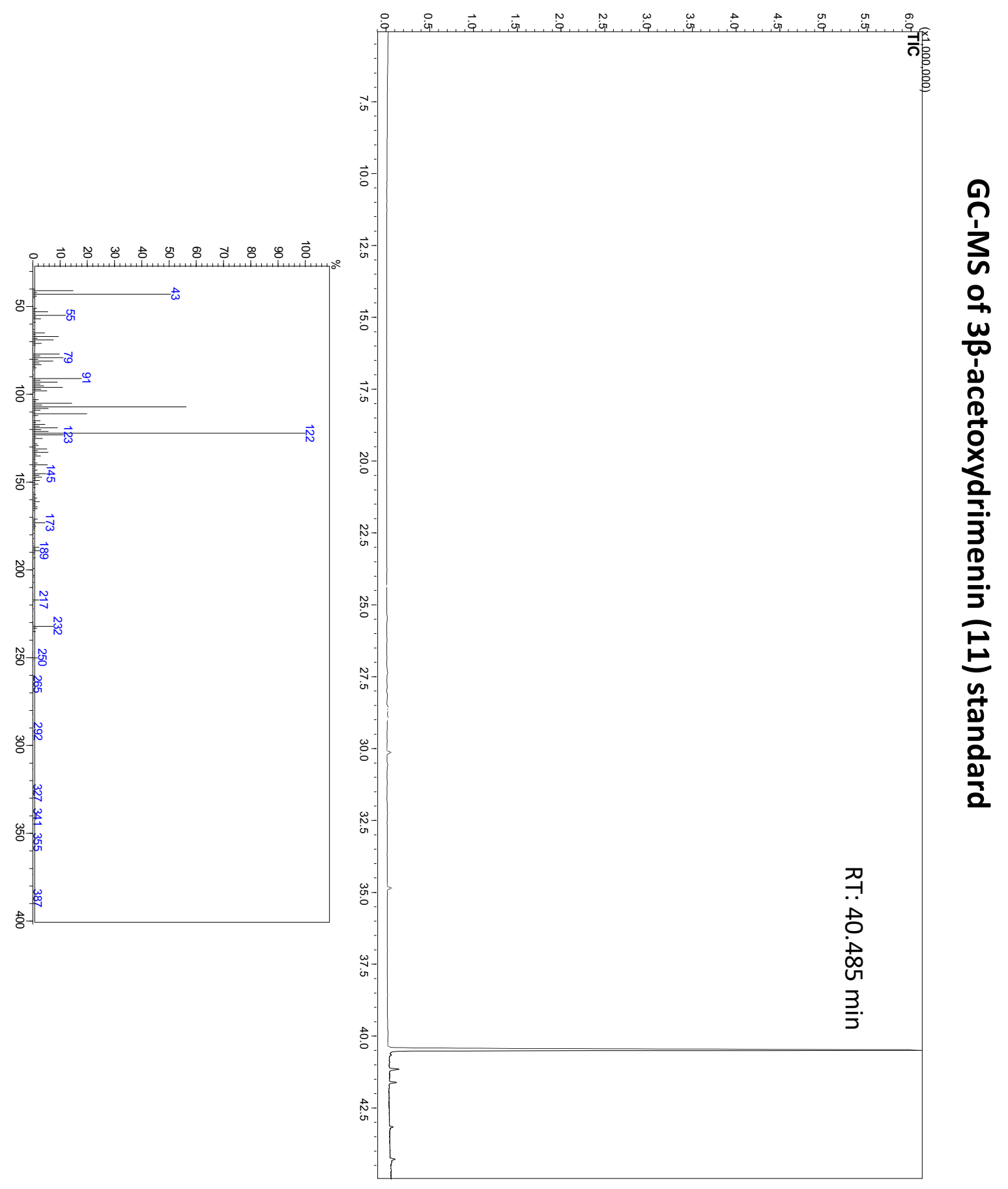




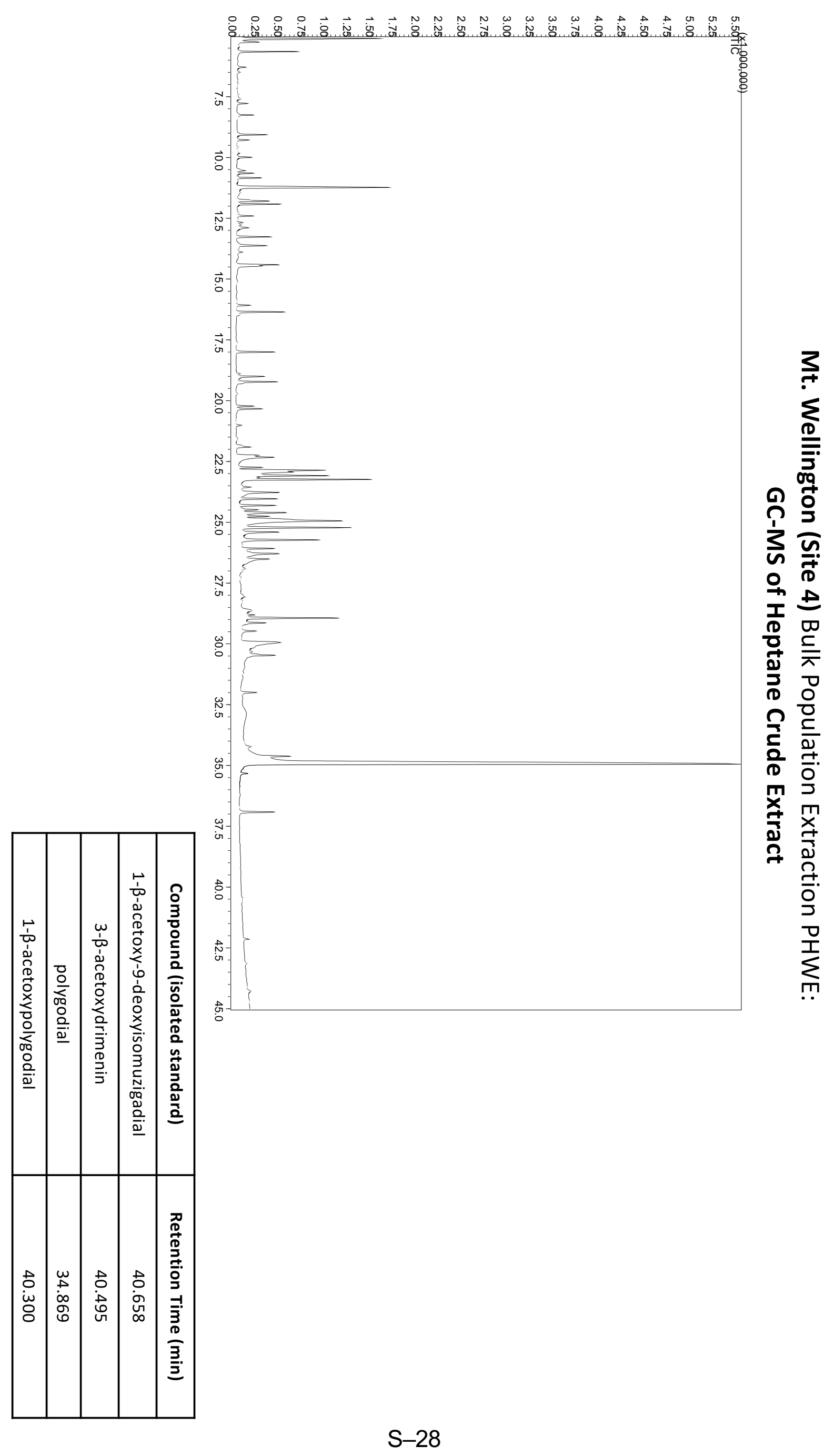




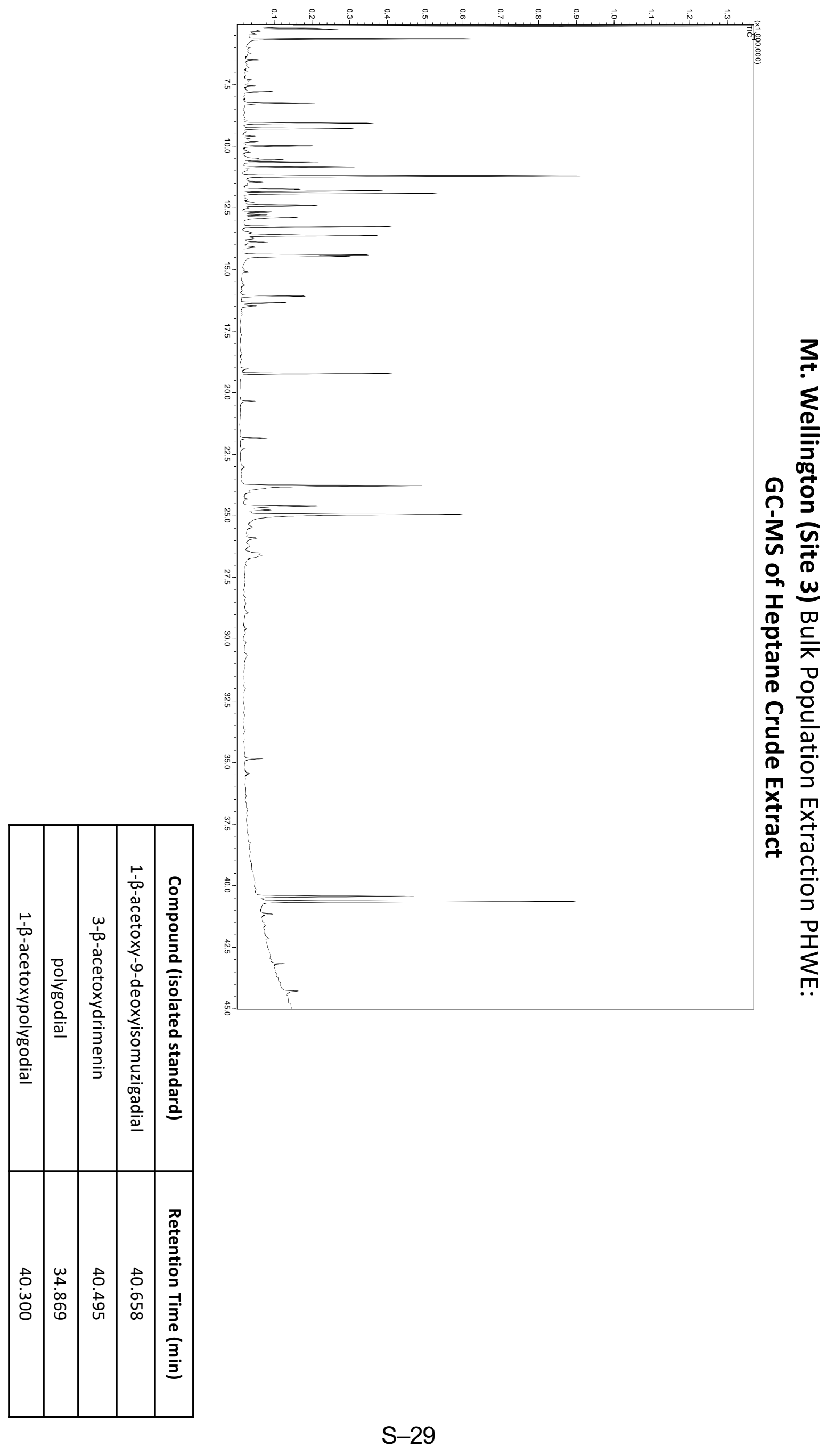




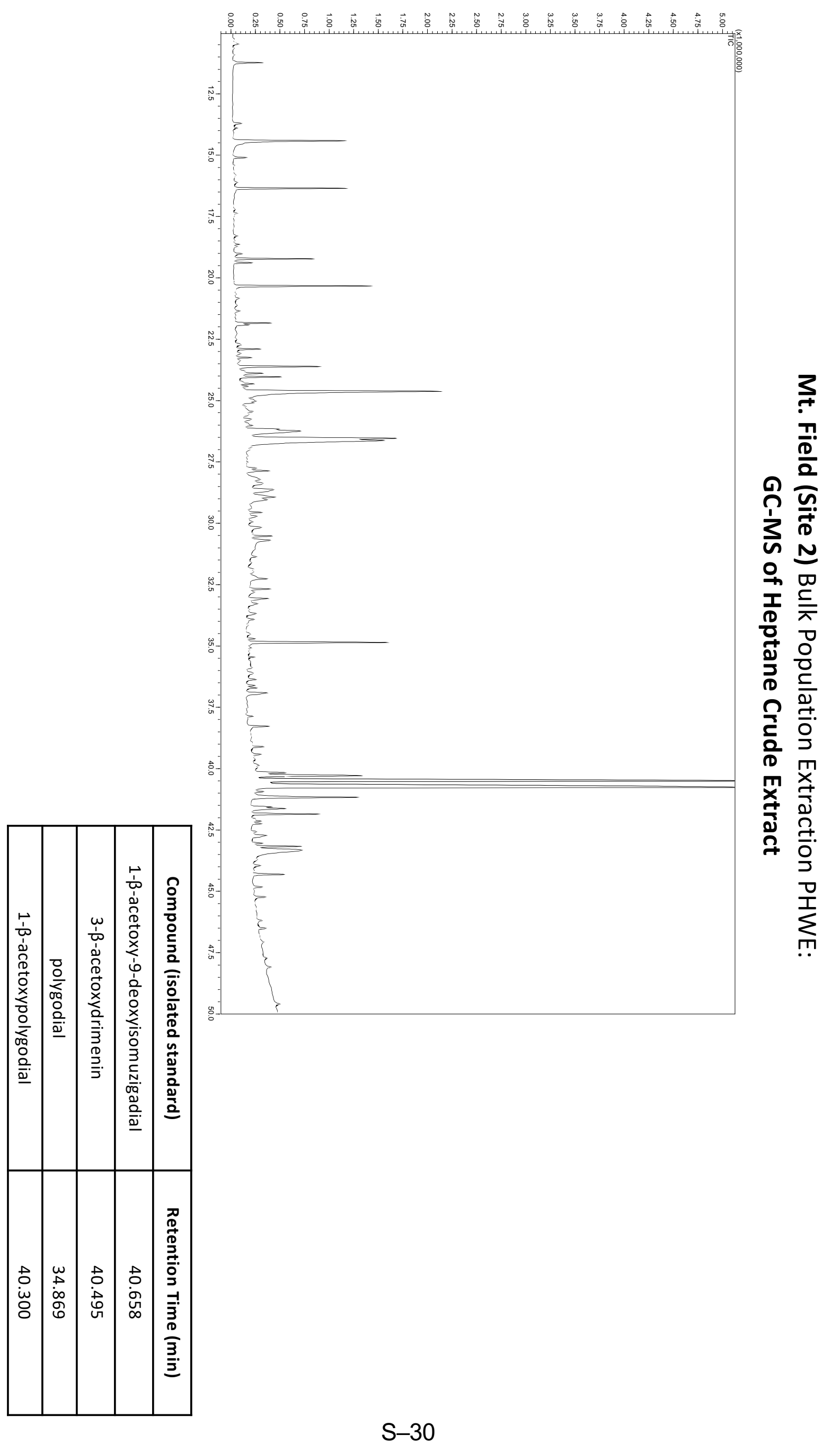




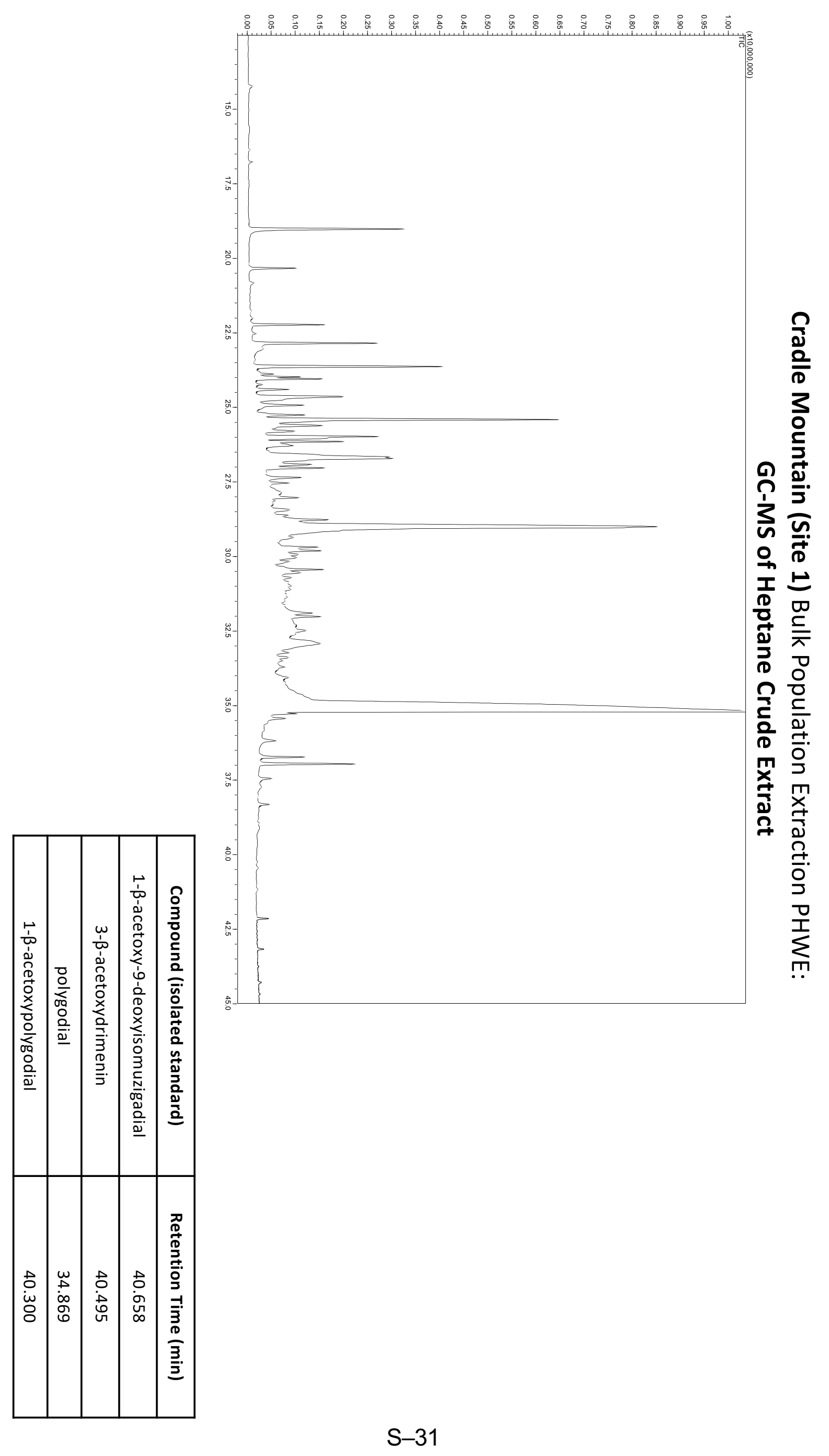

